TRANSACTIONS OF THE

AMERICAN MATHEMATICAL SOCIETY

Volume 348, Number 12, December 1996, Pages 4857-4882

S 0002-9947(96)01753-9

\title{
ON EXTENSION OF COCYCLES TO NORMALIZER ELEMENTS, OUTER CONJUGACY, AND RELATED PROBLEMS
}

\author{
ALEXANDRE I. DANILENKO AND VALENTIN YA. GOLODETS
}

\begin{abstract}
Let $T$ be an ergodic automorphism of a Lebesgue space and $\alpha$ a cocycle of $T$ with values in an Abelian locally compact group $G$. An automorphism $\theta$ from the normalizer $N[T]$ of the full group $[T]$ is said to be compatible with $\alpha$ if there is a measurable function $\varphi: X \rightarrow G$ such that $\alpha\left(\theta x, \theta T \theta^{-1}\right)=-\varphi(x)+\alpha(x, T)+\varphi(T x)$ at a.e. $x$. The topology on the set $D(T, \alpha)$ of all automorphisms compatible with $\alpha$ is introduced in such a way that $D(T, \alpha)$ becomes a Polish group. A complete system of invariants for the $\alpha$-outer conjugacy (i.e. the conjugacy in the quotient group $D(T, \alpha) /[T]$ ) is found. Structure of the cocycles compatible with every element of $N[T]$ is described.
\end{abstract}

\section{INTRODUCTION}

0.1. The study of cocycles of dynamical systems is an important trend in modern ergodic theory. Cocycles retain essential information about the dynamical system as group representations do about group structure. They have numerous and diverse applications. So, cocycles arise naturally when studying extensions of ergodic group actions and equivalence relations [FSZ], [Z], in the representation theory, etc. [M1], $[\mathrm{K}],[\mathrm{G}]$. And one of the most important aspects explaining a vigorous interest in cocycles is G. Mackey's construction of a group action associated with cocycles [M2] which generalizes the well known notion of a flow built under function.

0.2. Let $\alpha$ be a cocycle of an ergodic dynamical system $(X, \mu, \Gamma)$ with values in a locally compact second countable Abelian group $G$. We say that an element $\theta$ of the normalizer $N[\Gamma]$ of the full group $[\Gamma]$ is compatible with $\alpha$ if the cocycle $\alpha \circ \theta$ is cohomologous to $\alpha$ (this implies that $\alpha$ can be extended to the group $\Gamma_{\theta}$ generated by $\Gamma$ and $\theta$ as a cocycle with values in an extension of $G)$. Denote by $D(\Gamma, \alpha)$ the group of all $\alpha$-compatible automorphisms. $D(\Gamma, \alpha)$ is an $\alpha$-analogue of $N[\Gamma]$ and precisely coincides with it when $\alpha$ is a coboundary or the Radon-Nikodym cocycle. This group retains essential information on $\alpha$. So, for example, if $D(\Gamma, \alpha)=[\Gamma]$ then $\alpha$ is transient, or if $\Gamma$ is of type $I I$ and $N[\Gamma]=D(\Gamma, \alpha)$ then $\alpha$ is a coboundary. In the paper the topological and algebraic structures of $D(\Gamma, \alpha)$ are studied. It is proved that $D(\Gamma, \alpha)$ is a Polish group with respect to some metric $d_{\alpha}$ which is determined explicitly. The group of approximately inner automorphisms, i.e. the

Received by the editors January 4, 1995.

1991 Mathematics Subject Classification. Primary 46L55; Secondary 28D15, 28D99.

Key words and phrases. Ergodic dynamical system, cocycle, outer conjugacy.

The work was supported in part by the International Science Foundation Grant No U2B000. 
closure of $[\Gamma]$ in $d_{\alpha}$, is described. The continuous $\alpha$-fundamental homomorphism from $D(\Gamma, \alpha)$ into the centralizer $C\left(W_{\alpha}\right)$ of the action $W_{\alpha}(G)$ associated with $\alpha$ is constructed. It is established that the quotient group $D(\Gamma, \alpha) /[\Gamma]$ is isomorphic to $C\left(W_{\alpha}\right) / W_{\alpha}(G)$ when $\alpha$ is transient. Moreover, we obtain a simple complete system of invariants for the $\alpha$-outer conjugacy of automorphisms compatible with $\alpha$, i.e. the conjugacy of their projections in $D(\Gamma, \alpha) /[\Gamma]$. Notice that this equivalence relation is a natural generalization of the classical outer conjugacy in $N[\Gamma]$. However, as it turns out the above system contains an invariant that has no analogy in the classical case (see $\S 1.4$ and Theorems 3.1 and 5.8). Thus, one more problem of the "relative" classification is solved: the classification of automorphisms with respect to an amenable equivalence relation and a cocycle defined on it. In addition we consider a problem of the "converse sort". Namely, given an abstract group $D$, $[\Gamma] \subset D \subset N[\Gamma]$, what can be said about the cocycles $\alpha$ with $D(\Gamma, \alpha)=D$ ? We solve only a particular case of this extremely hard problem: the complete weak classification of cocycles $\alpha$ of the Radon-Nikodym type is obtained, i.e. with $D(\Gamma, \alpha)=N[\Gamma]$, and their structure is described in a transparent way.

When $\alpha$ is a coboundary the above results are well known [CK], [BG1], [H], [HO]. But in the general case (for an arbitrary $\alpha$ ) one encounters certain new problems such as the possible nondivisibility of $G$ (this fact puts an obstacle for the extension of a cocycle to outer periodic automorphisms). Moreover, transient cocycles exist, the extension of a cocycle to an automorphism compatible with it is not unique, etc.

The theory developed in this work turned out to be a useful tool in the paper [GS2] devoted to classification of cocycles of amenable equivalence relations, and in [GD] where the joint actions of two ergodic actions of an Abelian group were studied.

0.3. The outline of the paper is as follows. Section 1 contains some background in the ergodic theory. The basic concepts of the present paper are also formulated here. Section 2 is devoted to the study of some topological properties of $D(\Gamma, \alpha)$ and introduces the $\alpha$-fundamental homomorphism. In Section 3 regular cocycles are considered. We describe the group of approximately inner automorphisms, structure of automorphisms compatible with $\alpha$ and find simple necessary and sufficient conditions for two automorphisms compatible with $\alpha$ to be $\alpha$-outer conjugate. The same problems are solved for nonregular cocycles when the actions associated them are free (Section 4) and nonfree (Section 5). And in the last section we study structure of cocycles which satisfy the property $D(\Gamma, \alpha)=N[\Gamma]$ and prove the results announced in [Da2].

We thank the referee for valuable comments.

\section{Preliminaries. Extension of COCyCle to nORMalizer elements}

One can find a detailed outline and discussion of the results stated below in $[\mathrm{Kr}]$, $[\mathrm{HO}],[\mathrm{S}],[\mathrm{BG} 3],[\mathrm{H}]$.

1.1. Let $(X, \mathcal{B}, \mu)$ be a non-atomic Lebesgue space, $\operatorname{Aut}(X, \mu)$ the set of all its automorphisms, i.e. one-to-one bimeasurable nonsingular transformations of $X$, and let $\Gamma$ be a countable ergodic subgroup of $\operatorname{Aut}(X, \mu)$. We shall assume that $\Gamma$ acts freely. The set $\{t \in \operatorname{Aut}(X, \mu) \mid t x \in \Gamma x$ for a.e. $x\}$ is called the full group $[\Gamma]$ of automorphisms of $(X, \mathcal{B}, \mu)$ generated by $\Gamma$. If there is a single transformation 
$T$ such that $[\Gamma]=\left[\left\{T^{n} \mid n \in \mathbb{Z}\right\}\right]$ then $\Gamma$ is called approximately finite (a.f.). An ergodic group $\Gamma$ is said to be type $I I_{1}\left(I I_{\infty}\right)$ if there exists a finite (infinite $\sigma$-finite) $\Gamma$-invariant measure, equivalent to $\mu$. Otherwise $\Gamma$ is said to be type III. if

A quadruple $\xi=(A, \Xi, A(),. \gamma(.,)$.$) is called a \Gamma$-array on the set $A \in \mathcal{B}, \mu(A)>0$,

(i) $\Xi$ is a finite set,

(ii) $\bigcup_{i \in \Xi} A(i)=A, A(i) \cap A(j)=\emptyset$ for all $i \neq j, \mu(A(i))>0$,

(iii) $\gamma(i, j)$ is a nonsingular isomorphism $A(j) \rightarrow A(i)$ such that $\gamma(i, j) x \in \Gamma x$ for a.e. $x \in A(j)$ and $\gamma(k, j) \gamma(j, i)=\gamma(k, i), \gamma(i, i)=\operatorname{id}_{A(i)}$ for all $i, j, k \in \Xi$.

By $\mathcal{G}(\xi)$ we denote the (finite) group of automorphisms of $A$ whose restrictions to every subset $A(i)$ coincide with some $\gamma(i, j), i, j \in \Xi$, and by $\mathcal{P}(\xi)$ the collection of the sets $\bigcup_{i \in \Delta} A(i)$, where $\Delta$ is an arbitrary subset of $\Xi$.

Let $\xi_{1}=(A, \Xi, A(),. \gamma(.,)$.$) and \xi_{2}=\left(A\left(i_{0}\right), \Omega, B(),. \delta(.,).\right)$ be two $\Gamma$-arrays, $i_{0} \in \Xi$. By the refinement of $\xi_{1}$ by $\xi_{2}$ we mean the $\Gamma$-array

$$
\xi_{1} \times \xi_{2}=(A, \Xi \times \Omega, C(., .), \tau(., . ; ., .)),
$$

where $C(i, n)=\gamma\left(i, i_{0}\right) B(n)$ and $\tau\left(i_{1}, n_{1} ; i, n\right)=\gamma\left(i_{1}, i_{0}\right) \delta\left(n_{1}, n\right) \gamma\left(i_{0}, i\right)$ for all $i, i_{0}, i_{1} \in \Xi, n, n_{1} \in \Omega$.

We denote by $N[\Gamma]$ the normalizer of $[\Gamma]$ in $\operatorname{Aut}(X, \mu)$, i.e.

$$
N[\Gamma]=\left\{\theta \in \operatorname{Aut}(X, \mu) \mid \theta[\Gamma] \theta^{-1}=[\Gamma]\right\} .
$$

The least positive integer $p$ such that $\theta^{p} \in[\Gamma]$ is called the outer period $(p(\theta))$ of $\theta$. If such an integer does not exist, we set $p(\theta)=0$. As usual, $C(\Gamma)$ stands for the centralizer of $\Gamma$, i.e. $C(\Gamma)=\{\theta \in \operatorname{Aut}(X, \mu) \mid \theta \gamma=\gamma \theta$ for all $\gamma \in \Gamma\}$. It is clear that $C(\Gamma) \subset N[\Gamma]$.

1.2. Let $G$ be a locally compact second countable (l.c.s.c.) group. A measurable map $\alpha: X \times \Gamma \rightarrow G$ is said to be a cocycle of the dynamical system (d.s.) $(X, \mathcal{B}, \mu, \Gamma)$ with values in $G$ if $\alpha\left(x, \gamma_{1} \gamma_{2}\right)=\alpha\left(\gamma_{2} x, \gamma_{1}\right) \alpha\left(x, \gamma_{2}\right)$ for all $\gamma_{i} \in \Gamma$ at a.e. $x \in X$. The set of all cocycles will be denoted by $Z^{1}(X \times \Gamma, G)$. It is easy to extend $\alpha$ to $[\Gamma]$. Two cocycles $\alpha$ and $\beta$ are said to be cohomologous if $\beta(x, \gamma)=\phi(\gamma x) \alpha(x, \gamma) \phi(x)^{-1}$ for some measurable function $\phi: X \rightarrow G$ and all $\gamma \in \Gamma$ at a.e. $x$. By a coboundary we mean a cocycle which is cohomologous to the trivial one.

Let us consider two d.s. $\left(X_{i}, \mathcal{B}_{i}, \mu_{i}, \Gamma_{i}\right)$ and their cocycles $\alpha_{i} \in Z^{1}\left(X_{i} \times \Gamma_{i}, G\right)$, $i=1,2$. Two pairs $\left(\Gamma_{1}, \alpha_{1}\right)$ and $\left(\Gamma_{2}, \alpha_{2}\right)$ are said to be weakly equivalent if there is an isomorphism $\phi: X_{1} \rightarrow X_{2}$ such that $\left[\Gamma_{1}\right]=\phi^{-1}\left[\Gamma_{2}\right] \phi$ and the cocycles $\phi \circ \alpha_{1}$ and $\alpha_{2}$ are cohomologous. The cocycle $\phi \circ \alpha_{1}$ is defined as follows: $\phi \circ \alpha_{1}\left(x_{2}, \gamma_{2}\right)=$ $\alpha_{1}\left(\phi^{-1} x_{2}, \phi^{-1} \gamma_{2} \phi\right)$ for all $\gamma_{2} \in \Gamma_{2}$ at a.e. $x_{2} \in X_{2}$.

Let $\tau$ be the shift on the group $\mathbb{Z}, \hat{\Gamma}=\Gamma \times\left\{\tau^{n} \mid n \in \mathbb{Z}\right\}$ and $\hat{\alpha}\left(x, k, \gamma \times \tau^{n}\right)=$ $\alpha(x, \gamma)$ for all $n \in \mathbb{Z}, \gamma \in \Gamma$ at all $(x, k) \in X \times \mathbb{Z}$. The pair $(\hat{\Gamma}, \hat{\alpha})$ is called the countable extension of $(\Gamma, \alpha)$. We say that $\left(\Gamma_{1}, \alpha_{1}\right)$ and $\left(\Gamma_{2}, \alpha_{2}\right)$ are stably weak equivalent if their countable extensions are weakly equivalent.

Let $\mu_{G}$ be a left Haar measure for $G$. Then one can define the actions $V$ of $G$ and $V_{\alpha}$ of $\Gamma$ on $\left(X \times G, \mu \times \mu_{G}\right)$ as follows:

$$
V(g)(x, h)=\left(x, h g^{-1}\right), \quad V_{\alpha}(\gamma)(x, h)=(\gamma x, \alpha(x, \gamma) h) .
$$

Since $V_{\alpha}(\gamma) V(g)=V(g) V_{\alpha}(\gamma)$ for every $g \in G, \gamma \in \Gamma$, it follows that $V$ induces a new action of $G$ on the space $(\Omega, \nu)$ of $V_{\alpha}$-ergodic components. We call it the action associated with $(\Gamma, \alpha)$ or the Mackey action and denote by $W_{\alpha}$. If $\Gamma$ is of type $I I I$, 
then we shall consider the "double" cocycle $\alpha_{0}=(\alpha, \rho) \in Z^{1}(X \times \Gamma, G \times \mathbb{R})$ as well as $\alpha$, where $\rho$ is the Radon-Nikodym cocycle, i.e. $\rho(x, \gamma)=\log \frac{d \mu \circ \gamma}{d \mu}(x)$.

Let $G$ be an Abelian group and $\bar{G}$ the one-point compactification of $G$. An element $g \in \bar{G}$ is called an essential value of $\alpha$ if for every set $B \in \mathcal{B}, \mu(B)>0$, and each neighborhood $U$ of $g$ in $\bar{G}$ there exist a subset $B_{1} \subset B, \mu\left(B_{1}\right)>0$, and an automorphism $\gamma \in \Gamma$ such that $\gamma B_{1} \subset B$ and $\alpha(x, \gamma) \in U$ for a.e. $x \in B_{1}$. By $\bar{r}(\Gamma, \alpha)$ we denote the set of all essential values of $\alpha$. Let $r(\Gamma, \alpha)=\bar{r}(\Gamma, \alpha) \cap G$. The set $r(\Gamma, \alpha)$ is a closed subgroup in $G$ and is invariant (as well as $\bar{r}(\Gamma, \alpha)$ ) under the stably weak equivalence.

Definition 1.1. An automorphism $\theta \in N[\Gamma]$ is called compatible with $\alpha$ (or admissible for $\alpha$ [BGD]) if $\alpha$ and $\theta^{-1} \circ \alpha$ are cohomologous, i.e. there is a measurable function $\varphi: X \rightarrow G$ such that

$$
\alpha\left(\theta x, \theta \gamma \theta^{-1}\right)=\varphi(\gamma x) \alpha(x, \gamma) \varphi(x)^{-1} \quad \text { for all } \gamma \in \Gamma \text { at a.e. } x \in X .
$$

By $\alpha(\theta)$ we denote the set of all functions $\varphi$ satisfying (1) and by $D(\Gamma, \alpha)$ the set of all automorphisms compatible with $\alpha$. Obviously, $D(\Gamma, \alpha)$ is a subgroup of $N[\Gamma]$. Since for each automorphism $\delta \in[\Gamma]$ we have

$$
\alpha\left(\delta x, \delta \gamma \delta^{-1}\right)=\alpha(\gamma x, \delta) \alpha(x, \gamma) \alpha\left(\delta x, \delta^{-1}\right)=\alpha(\gamma x, \delta) \alpha(x, \gamma) \alpha(x, \delta)^{-1}
$$

for all $\gamma \in \Gamma$ at a.e. $x$, it follows that $[\Gamma] \subset D(\Gamma, \alpha)$. If $\zeta \in N[\Gamma]$, then $D(\Gamma, \zeta \circ \alpha)=$ $\zeta D(\Gamma, \alpha) \zeta^{-1}$. If $\alpha$ is a coboundary, then $D(\Gamma, \alpha)=N[\Gamma]$.

Example 1.2. Let $X=[0,1) \subset \mathbb{R}, \mu$ be the Lebesgue measure on $X,\left\{t_{j}\right\}_{j=0}^{\infty}$ a sequence of rationally independent irrational numbers, $G$ an Abelian l.c.s.c. group and $G_{d}=\left\{g_{j}\right\}_{j=1}^{\infty}$ a countable dense subgroup of $G$. Let us consider automorphisms $\gamma_{j}$ and $\theta_{j p}$ on $(X, \mu): \gamma_{j} x=x+t_{j}(\bmod 1), \theta_{j p} x=x+t_{j} p^{-1}(\bmod 1)$. By $\Gamma$ we denote the group generated by $\gamma_{j}, j \in \mathbb{N}$. Obviously, $\gamma_{0}, \theta_{j p} \in N[\Gamma]$ for all $j, p \in \mathbb{N}$. We define the cocycle $\alpha$ by setting $\alpha\left(x, \gamma_{j}\right)=g_{j}, j \in \mathbb{N}$. It is easy to see that $\gamma_{0}$ and $\theta_{j p}$ are compatible with $\alpha$, and $p\left(\gamma_{0}\right)=0, p\left(\theta_{j p}\right)=p$.

Example 1.3. Let $(X, \mu)=\prod_{i=1}^{\infty}\left(X_{i}, \mu_{i}\right)$, where $X_{i}=\{0,1\}$ and $\mu_{i}(0)=\mu_{i}(1)=$ $2^{-1}$. An Abelian measure preserving group $\Gamma$ is generated by the automorphisms $\gamma_{j}, j \in \mathbb{N}$, given by $\left(\gamma_{j} x\right)_{i}=x_{i}+\delta_{i j}(\bmod 2)$ for every sequence $x$ of 0 's and 1 's. A cocycle $\alpha$ of the d.s. $(X, \mu, \Gamma)$ with values in $\mathbb{Z}$ is determined as follows: $\alpha\left(x, \gamma_{j}\right)=(-1)^{x_{j}} j$. We define the automorphism $\theta$ by setting $(\theta x)_{i}=x_{i}+1$ $\bmod 2, i \in \mathbb{N}$. Evidently, $\theta \in N[\Gamma]$. If $\theta$ is compatible with $\alpha$ and $\varphi \in \alpha(\theta)$, then it follows from (1) that $\varphi(x)-\varphi\left(\gamma_{j} x\right)=(-1)^{x_{j}} 2 j$ for all $j \in \mathbb{N}$ at a.e. $x$. Take a number $N$ such that $\mu(\{x \in X|| \varphi(x) \mid<N\})>2^{-1}$. Then for every $j \in \mathbb{N}$ we have $\left|\varphi(x)-\varphi\left(\gamma_{j} x\right)\right|<2 N$ on some set of positive measure, a contradiction. So, $\theta$ is not compatible with $\alpha$. We have established also that $\alpha$ is not a coboundary.

Let $H$ be a central closed subgroup of $G$ and $\alpha(x, \gamma) \in H$ for all $\gamma \in \Gamma$ at a.e. $x$. Then we consider the cocycle $\alpha_{H} \in Z^{1}(X \times \Gamma, H)$ given by $\alpha_{H}(x, \gamma)=\alpha(x, \gamma)$.

Proposition 1.4. $D\left(\Gamma, \alpha_{H}\right)=D(\Gamma, \alpha)$.

Proof. Let $\theta \in D(\Gamma, \alpha)$ and $\varphi \in \alpha(\theta)$. Since $\varphi(\gamma x) \varphi(x)^{-1} \in H$ for all $\gamma \in \Gamma$ at a.e. $x$ and $\Gamma$ is ergodic, we have $\varphi(x)=g \psi(x)$ at a.e. $x$ for some function $\psi: X \rightarrow H$ and some element $g \in G$. Evidently, $\theta \in D\left(\Gamma, \alpha_{H}\right)$ and $\psi \in \alpha_{H}(\theta)$. It is trivial that $D\left(\Gamma, \alpha_{H}\right) \subset D(\Gamma, \alpha)$. 
Definition 1.5. Two $\alpha$-compatible automorphisms $\theta_{1}$ and $\theta_{2}$ are said to be $\alpha$ outer conjugate if there exist automorphisms $\zeta \in D(\Gamma, \alpha)$ and $t \in[\Gamma]$ such that $\zeta \theta_{1} \zeta^{-1}=\theta_{2} t$.

Obviously, the $\alpha$-outer conjugacy is an equivalence relation on $D(\Gamma, \alpha)$.

Let an automorphism $\theta \in D(\Gamma, \alpha)$ and a function $\varphi \in \alpha(\theta)$. We define an automorphism $\theta_{\varphi}$ of $\left(X \times G, \mu \times \mu_{G}\right)$ by setting $\theta_{\varphi}(x, g)=(\theta x, \varphi(x) g)$. Then $\theta_{\varphi} \in N\left[V_{\alpha}(\Gamma)\right] \cap C(V)$ (see $\S 1.2$ ), where $C(V)$ is the centralizer of $V(G)$ in $\operatorname{Aut}\left(X \times G, \mu \times \mu_{G}\right)$. Hence, $\theta_{\varphi}$ induces an automorphism of $(\Omega, \nu)$ (the space of $V_{\alpha}(\Gamma)$-ergodic components). We denote it by $\Psi_{\alpha}(\theta, \varphi)$. Evidently, $\Psi_{\alpha}(\theta, \varphi) \in$ $C\left(W_{\alpha}\right)$.

1.3. Below in this paper we assume that $G$ is an Abelian group. Then for every two functions $\varphi, \psi$ from $\alpha(\theta)$ there exists an element $g \in G$ such that $\varphi(x)-\psi(x)=g$ for a.e. $x$, i.e. (1) determines $\varphi$ up to an additive constant. Therefore, $\Psi_{\alpha}(\theta, \varphi)=$ $\Psi_{\alpha}(\theta, \psi) W_{\alpha}(g)$ and it is natural to define a map $\Phi_{\alpha}: D(\Gamma, \alpha) \rightarrow C\left(W_{\alpha}\right) / W_{\alpha}(G)$ by $\Phi_{\alpha}(\theta)=\Psi_{\alpha}(\theta, \varphi) W_{\alpha}(G)$. We call it the $\alpha$-fundamental homomorphism.

Let $W_{\alpha_{0}}$ be the action of $G \times \mathbb{R}$ associated with $\left(\Gamma, \alpha_{0}\right)$ on the space $\left(\Omega_{0}, \nu_{0}\right)$. We consider the action $W_{\alpha_{0}}^{r}$ of $\mathbb{R}$ given by $W_{\alpha_{0}}^{r}(t)=W_{\alpha_{0}}(0, t)$. The $W_{\alpha_{0}}^{r}$-ergodic decomposition of $\left(\Omega_{0}, \nu_{0}\right)$ induces the map $\tau: C\left(W_{\alpha_{0}}\right) \rightarrow C\left(W_{\alpha}\right)$ which can be extended to the homomorphism $\tau_{0}: C\left(W_{\alpha_{0}}\right) / W_{\alpha_{0}}(G, 0) \rightarrow C\left(W_{\alpha}\right) / W_{\alpha}(G)$ and the diagram

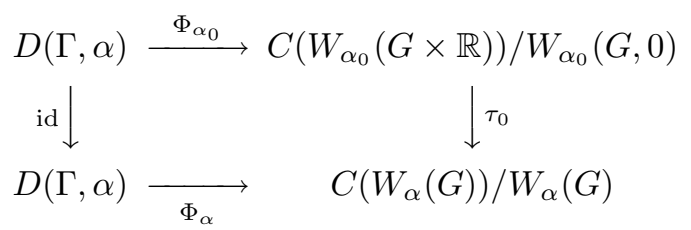

is commutative.

If $\theta \in D(\Gamma, \alpha)$, one can extend $\alpha$ to a cocycle of the group $\Gamma_{\theta}$ generated by $\Gamma$ and $\theta$ and whose values are in an extension of $G$. Let $\varphi \in \alpha(\theta)$. We separately consider two cases.

1. $\theta$ is outer aperiodic, i.e. $p(\theta)=0($ see $\S 1.2)$. Set

$$
\left\{\begin{array}{l}
\tilde{\alpha}(x, \gamma)=(\alpha(x, \gamma), 0), \\
\tilde{\alpha}(x, \theta)=(\varphi(x), 1),
\end{array}\right.
$$

where the right-hand side belongs to $G \times \mathbb{Z}$. Obviously, $\tilde{\alpha} \in Z^{1}\left(X \times \Gamma_{\theta}, G \times \mathbb{Z}\right)$.

2. $\theta$ is outer periodic, i.e. $p=p(\theta)>0$. It follows from (1) that $\varphi(x)+\varphi(\theta x)+$ $\cdots+\varphi\left(\theta^{p-1} x\right) \in \alpha\left(\theta^{p}\right)$. On the other hand, the function $X \ni x \mapsto \alpha\left(x, \theta^{p}\right)$ belongs to $\alpha\left(\theta^{p}\right)$. Therefore, there exists an element $g=g(\theta, \varphi) \in G$ with

$$
\alpha\left(x, \theta^{p}\right)-\varphi(x)-\varphi(\theta x)-\cdots-\varphi\left(\theta^{p-1} x\right)=g
$$

for a.a. $x$. Notice that if another function $\psi$ belongs to $\alpha(\theta)$ then $\varphi(x)-\psi(x)=p g_{1}$ at a.e. $x$ for some $g_{1} \in G$. The Cartesian product $G \times\{0,1, \ldots, p-1\}$ is an Abelian l.c.s.c. group if one introduces addition as

$$
\left(g_{1}, n_{1}\right)+\left(g_{2}, n_{2}\right)=\left(g_{1}+g_{2}+g\left[\left(n_{1}+n_{2}\right) p^{-1}\right], n_{1} \dot{+} n_{2}\right),
$$

where the sign $\dot{+}$ means addition $\bmod p$ and [.] the integer part. We denote this group by $G(g, p)$. Then $G$ is contained in $G(g, p)$ as a closed subgroup of all elements of the form $(g, 0)$. The element $g=g(\theta, \varphi)$ can be divided by $p$ 
in $G(g, p)$, since $p(0,1)=(g, 0)$. Therefore, (3) correctly determines a cocycle $\tilde{\alpha} \in Z^{1}\left(X \times \Gamma_{\theta}, G(g, p)\right)$.

Remark 1.6. In the case (i) (or (ii) if $g=p g_{1}$ for some $g_{1} \in G$ ), one can define an extension $\alpha^{+} \in Z^{1}\left(X \times \Gamma_{\theta}, G\right)$ of $\alpha$ as follows: $\alpha^{+}(x, \theta)=\varphi(x)$ (or $\alpha^{+}(x, \theta)=$ $\varphi(x)+g_{1}$ respectively). But the extension $\tilde{\alpha}$ is more useful to study the $\alpha$-outer conjugacy (see Lemma 3.2 below).

It is easy to check that if two $\alpha$-compatible automorphisms $\theta_{1}$ and $\theta_{2}$ are $\alpha$-outer conjugate, then $g\left(\theta_{1}, \varphi_{1}\right)=g\left(\theta_{2}, \varphi_{2}\right)$ for some functions $\varphi_{i} \in \alpha\left(\theta_{i}\right)$ (if $p(\theta)=0$ we put $g(\theta, \varphi)=0)$. In Example 1.2, $g\left(\theta_{j p}, \varphi\right)=g_{j}$ for $\varphi=0$ and every $j \in \mathbb{N}$.

\section{Topology on $D(\Gamma, \alpha)$}

Throughout this section we assume that $\mu(X)=1$ and $\tau$ is a bounded invariant metric on $G$ compatible with the l.c.s.c. topology. A sequence $\left\{\phi_{n}\right\}_{n=1}^{\infty}$ of measurable functions $\phi_{n}: X \rightarrow G$ is said to converge in measure to a measurable function $\phi: X \rightarrow G$ if $\mu\left(\left\{x \mid \tau\left(\phi_{n}(x), \phi(x)\right)>\delta\right\}\right) \rightarrow 0$ as $n \rightarrow \infty$ for every $\delta>0$. We recall the definition of the Polish topology on $N[\Gamma]$ introduced in [HO] (cf. [Da3]). Let $d_{u}$ denote the uniform distance on $[\Gamma]$, i.e.

$$
d_{u}\left(\delta_{1}, \delta_{2}\right)=\mu\left(\left\{x \in X \mid \delta_{1} x \neq \delta_{2} x\right\}\right)+\mu\left(\left\{x \in X \mid \delta_{1}^{-1} x \neq \delta_{2}^{-1} x\right\}\right),
$$

and $d_{w}$ a metric being compatible with the weak topology on $\operatorname{Aut}(X, \mu)$. If we enumerate elements of $\Gamma$ as $\left\{\gamma_{k} \mid k \in \mathbb{N}\right\}$ then $d$ given by

$$
d\left(\theta_{1}, \theta_{2}\right)=d_{w}\left(\theta_{1}, \theta_{2}\right)+\sum_{k=1}^{\infty} \frac{1}{2^{k}} d_{u}\left(\theta_{1} \gamma \theta_{1}^{-1}, \theta_{2} \gamma_{k} \theta_{2}^{-1}\right)
$$

is a distance on $N[\Gamma]$ giving a Polish topology. It is clear that this topology is stronger than the weak one. Now we define the $\alpha$-topology on $D(\Gamma, \alpha)$ via the $\alpha$-convergence as follows.

Definition 2.1. A sequence $\left\{\theta_{n}\right\}_{n=1}^{\infty}$ of $\alpha$-compatible automorphisms is said to $\alpha$-converge to an automorphism $\theta \in D(\Gamma, \alpha)$ if there are functions $\phi_{n} \in \alpha\left(\theta_{n}\right)$, $n \in \mathbb{N}$, and $\phi \in \alpha(\theta)$ such that $\left\{\phi_{n}\right\}_{n=1}^{\infty}$ converges in measure to $\phi$ and $\left\{\theta_{n}\right\}_{n=1}^{\infty}$ converges to $\theta$ in the metric $d$.

Let $\theta_{i} \in D(\Gamma, \alpha)$ and $\phi_{i} \in \alpha\left(\theta_{i}\right), i=1,2$. Put

$$
\tilde{d}_{\alpha}\left(\theta_{1}, \theta_{2}\right)=\inf _{g \in G} \int_{X} \tau\left(\phi_{1}(x), \phi_{2}(x)+g\right) d \mu(x)+d\left(\theta_{1}, \theta_{2}\right) .
$$

Theorem 2.2. The $\alpha$-topology on $D(\Gamma, \alpha)$ is Polish and compatible with the metric $\tilde{d}_{\alpha}$.

Proof. By $M(X, G)$ we denote the space of all measurable maps from $X$ to $G$. The metric $\lambda$ given by

$$
\lambda\left(f_{1}, f_{2}\right)=\int_{X} \tau\left(f_{1}(x), f_{2}(x)\right) d \mu(x)
$$

determines the topology of convergence in measure on $M(X, G)$. Let us prove that the subset

$$
L=\{(\theta, \phi) \mid \theta \in D(\Gamma, \alpha), \phi \in \alpha(\theta)\}
$$


is a closed subset of $N[\Gamma] \times M(X, G)$. Consider a pair $(\theta, \phi) \in \bar{L}$. There is a sequence $\left\{\left(\theta_{n}, \phi_{n}\right)\right\}_{n=1}^{\infty}$ of pairs from $L$ such that $d\left(\theta_{n}, \theta\right) \rightarrow 0$ and $\phi_{n}(x) \rightarrow \phi(x)$ as $n \rightarrow \infty$ for a.e. $x$. We shall assume below for simplicity's sake that $\Gamma$ is a.f. (for non a.f. groups the proof can be slightly changed in an obvious way). Denote by $T$ an ergodic transformation with $[T]=[\Gamma]($ see $\S 1)$. Then for every $n \in \mathbb{N}$

$$
\alpha\left(x, \theta_{n}^{-1} T \theta_{n}\right)=\phi_{n}(x)+\alpha\left(\theta_{n} x, T\right)-\phi_{n}\left(\theta_{n}^{-1} T \theta_{n} x\right) \quad \text { at a.a. } x .
$$

Let us choose a dense countable subset $\left\{g_{k}\right\}_{k=1}^{\infty}$ in $G$ and consider the positive bounded functions $\tau_{k}(x)=\tau\left(\alpha(x, T), g_{k}\right), k \in \mathbb{N}$. It follows from the weak convergence $\theta_{n} \rightarrow \theta$ that for every $k \in \mathbb{N}$

$$
\int_{X}\left|\tau_{k}\left(\theta_{n} x\right) \frac{d \mu \circ \theta_{n}}{d \mu}(x)-\tau_{k}(\theta) \frac{d \mu \circ \theta}{d \mu}(x)\right| d \mu(x) \rightarrow 0
$$

as $n \rightarrow \infty$. By the diagonal process one can choose a subsequence $\left\{n_{j}\right\}_{j=1}^{\infty}$ such that for every $k \in \mathbb{N}$

$$
\tau_{k}\left(\theta_{n_{j}} x\right) \frac{d \mu \circ \theta_{n_{j}}}{d \mu}(x) \rightarrow \tau_{k}(\theta x) \frac{d \mu \circ \theta}{d \mu}(x) \text { and } \frac{d \mu \circ \theta_{n_{j}}}{d \mu}(x) \rightarrow \frac{d \mu \circ \theta}{d \mu}(x)
$$

as $j \rightarrow \infty$ for a.e. $x$. Hence, there exists $\lim _{j \rightarrow \infty} \tau_{k}\left(\theta_{n_{j}} x\right)=\tau_{k}(\theta x)$ at a.e. $x$ for every $k \in \mathbb{N}$. We deduce from this that

$$
\alpha\left(\theta_{n_{j}} x, T\right) \rightarrow \alpha(\theta x, T)
$$

as $j \rightarrow \infty$ for a.e. $x$. Denote by $\gamma$ (resp. $\gamma_{j}$ ) the automorphism $\theta^{-1} T \theta$ (resp. $\left.\theta_{n_{j}}^{-1} T \theta_{n_{j}}, j \in \mathbb{N}\right)$. We have

$$
\tau\left(\phi_{n_{j}}\left(\gamma_{j} x\right), \phi(\gamma x)\right) \leq \tau\left(\phi_{n_{j}}\left(\gamma_{j} x\right), \phi_{n_{j}}(\gamma x)\right)+\tau\left(\phi_{n_{j}}(\gamma x), \phi(\gamma x)\right)=I_{j}^{1}+I_{j}^{2} .
$$

Put $A_{j}=\left\{x: \gamma_{j} x \neq \gamma x\right\}$. Since $d\left(\theta_{n_{j}}, \theta\right) \rightarrow 0$, we have $\mu\left(A_{j}\right) \rightarrow 0$ as $j \rightarrow \infty$. Without loss of generality, one can assume that $\sum_{j=1}^{\infty} \mu\left(A_{j}\right)<\infty$. Then, it follows from the Borel-Cantelli lemma that $\alpha\left(x, \gamma_{j}\right) \rightarrow \alpha(x, \gamma)$ and $I_{j}^{1} \rightarrow 0$ as $j \rightarrow \infty$ for a.e. $x$. Therefore, using (5), we pass to a limit in (4) as $n_{j} \rightarrow \infty$ :

$$
\alpha\left(x, \theta^{-1} T \theta\right)=\phi(x)+\alpha(\theta x, T)-\phi(T x)
$$

for a.e. $x$, since $I_{j}^{2} \rightarrow 0$. Thus, $(\theta, \phi) \in L$. Hence $L$ is a Polish space as a closed subset of $N[\Gamma] \times M(X, G)$. Consider the equivalence relation on $L:\left(\theta_{1}, \phi_{1}\right) \sim$ $\left(\theta_{2}, \phi_{2}\right)$ if $\theta_{1}=\theta_{2}$. One can naturally identify the set $L / \sim$ with $D(\Gamma, \alpha)$. Moreover, the Polish quotient topology on $L / \sim$ (or, equivalently, the $\alpha$-topology on $D(\Gamma, \alpha)$ ) is compatible with $\tilde{d}_{\alpha}$. Thus, the theorem is proved.

Now let $d_{\alpha}\left(\theta_{1}, \theta_{2}\right)=\tilde{d}_{\alpha}\left(\theta_{2}^{-1} \theta_{1}, 1\right)+\tilde{d}_{\alpha}\left(\theta_{1}^{-1} \theta_{2}, 1\right)+\tilde{d}_{\alpha}\left(\theta_{2} \theta_{1}^{-1}, 1\right)+\tilde{d}_{\alpha}\left(\theta_{1} \theta_{2}^{-1}, 1\right)$.

Theorem 2.3. $D(\Gamma, \alpha)$ is a Polish group with respect to the metric $d_{\alpha}$.

Proof. Let $\left\{\theta_{n}\right\}_{n=1}^{\infty}$ and $\left\{\zeta_{n}\right\}_{n=1}^{\infty}$ be two sequences of $\alpha$-compatible automorphisms converging to some $\theta$ and $\zeta$ from $D(\Gamma, \alpha)$ respectively. Let us choose functions $\phi_{n} \in \alpha\left(\theta_{n}\right), \psi_{n} \in \alpha\left(\zeta_{n}\right), \phi \in \alpha(\theta)$, and $\psi \in \alpha(\zeta)$ according to Definition 2.1. Put $\eta_{n}(x)=\phi_{n}(x)+\psi_{n}\left(\theta_{n} x\right)$ and $\eta(x)=\phi(x)+\psi(\theta x)$. Then $\eta_{n} \in \alpha\left(\zeta_{n} \theta_{n}\right)$ and $\eta \in \alpha(\zeta \theta)$. It follows from the definition of $d_{\alpha}$ that the multiplication in $D(\Gamma, \alpha)$ is continuous if $\eta_{n} \rightarrow \eta$ in measure as $n \rightarrow \infty$. We need an auxiliary lemma.

Lemma 2.4. If a sequence $\left\{\theta_{n}\right\}_{n=1}^{\infty}$ of automorphisms of $(X, \mu)$ weakly converges, then for every $\varepsilon>0$ there exist $\delta>0$ and $N \in \mathbb{N}$ such that $\mu\left(\theta_{n} A\right)<\varepsilon$ for every subset $A, \mu(A)<\delta$, and every $n \in \mathbb{N}$. 
Proof. Suppose $\left\{\theta_{n}\right\}_{n=1}^{\infty}$ weakly converges to an automorphism $\theta \in D(\Gamma, \alpha)$, but the conclusion of the lemma is not valid. Then there are a real number $\varepsilon>0$ and a sequence of measurable subsets $\left\{A_{n}\right\}_{n=1}^{\infty}$ such that $\mu\left(A_{n}\right)<2^{-n}$ and $\mu\left(\theta_{n} A_{n}\right)>\varepsilon$. We have

$$
\mu\left(\theta_{n} A_{n}\right)=\mu\left(\theta A_{n}\right)+\int_{A_{n}}\left(\frac{d \mu \circ \theta_{n}}{d \mu}(x)-\frac{d \mu \circ \theta}{d \mu}(x)\right) d \mu(x) .
$$

Since the absolute value of the second term in (6) is less than

$$
\int_{A_{n}}\left|\frac{d \mu \circ \theta_{n}}{d \mu}(x)-\frac{d \mu \circ \theta}{d \mu}(x)\right| d \mu(x) .
$$

and $\left\{\theta_{n}\right\}_{n=1}^{\infty}$ weakly converges to $\theta$, one can pass to the limit in (6): $\mu\left(\theta_{n} A_{n}\right) \rightarrow 0$ as $n \rightarrow \infty$, a contradiction.

Let us return to the proof of Theorem 2.3. For every positive integer $n$

$$
\tau\left(\eta_{n}(x), \eta(x)\right) \leq \tau\left(\phi(x), \phi_{n}(x)\right)+\tau\left(\psi_{n}\left(\theta_{n} x\right), \psi\left(\theta_{n} x\right)\right)+\tau\left(\psi\left(\theta_{n} x\right), \psi(\theta x)\right) .
$$

Now apply Lemma 2.4 to deduce that

$$
\mu\left(\left\{x \mid \tau\left(\psi_{n}\left(\theta_{n} x\right), \psi\left(\theta_{n} x\right)\right)>\delta\right\}\right)=\mu\left(\theta_{n}^{-1}\left\{x: \tau\left(\psi_{n}(x), \psi(x)\right)>\delta\right\}\right) \rightarrow 0
$$

as $n \rightarrow \infty$. It is easy to see that the other two terms on the right-hand side of (7) vanish also in measure as $n \rightarrow \infty$. The continuity of the inverse operation in $D(\Gamma, \alpha)$ is established in a similar way.

Proposition 2.5. Let a cocycle $\alpha \in Z^{1}(X \times \Gamma, G)$ take its values in a closed subgroup $H \subset G$ and the cocycle $\alpha_{H}$ be the same as in Proposition 1.4. Then the topology of $\alpha_{H}$-convergence on $D\left(\Gamma, \alpha_{H}\right)=D(\Gamma, \alpha)$ is equivalent to the topology of $\alpha$-convergence.

The proof is obvious.

Theorem 2.6. Let a sequence $\left\{\theta_{n}\right\}_{n=1}^{\infty}$ of $\alpha$-compatible automorphisms $\alpha$-converge to $\theta \in D(\Gamma, \alpha)$ and functions $\phi_{n} \in \alpha\left(\theta_{n}\right)$ and $\phi \in \alpha(\theta)$ be chosen according to Definition 2.1. Then the sequence $\left\{\Psi_{\alpha}\left(\theta_{n}, \phi_{n}\right)\right\}_{n=1}^{\infty}$ of automorphisms from $C\left(W_{\alpha}\right)$ weakly converges to $\Psi_{\alpha}(\theta, \phi)$.

This statement is similar to Theorem 4 from $[\mathrm{H}]$, where the Radon-Nikodym cocycle was considered. Therefore we state it here without proof.

\section{Automorphisms COMPATIBLE With REgUlAR COCYClES}

In this section we assume that $\Gamma$ is a.f. A cocycle $\alpha \in Z^{1}(X \times \Gamma, G)$ is called regular if the Mackey action $W_{\alpha}$ of $G$ is transitive [S], [BG3]. Then the stabilizer of $W_{\alpha}$ is equal to $r(\Gamma, \alpha)$ (see $\left.\S 1\right)$.

Theorem 3.1. Let a cocycle $\alpha$ be such that the "double" cocycle $\alpha_{0}=(\alpha, \rho)$ is regular. Then two $\alpha$-compatible automorphisms $\theta_{1}$ and $\theta_{2}$ are outer conjugate iff

$$
p\left(\theta_{1}\right)=p\left(\theta_{2}\right), g\left(\theta_{1}, \phi_{1}\right)=g\left(\theta_{2}, \phi_{2}\right), \text { and } \Psi_{\alpha_{0}}\left(\theta_{1},\left(\phi_{1}\right)_{0}\right)=\Psi_{\alpha_{0}}\left(\theta_{2},\left(\phi_{2}\right)_{0}\right)
$$

for some functions $\phi_{i} \in \alpha\left(\theta_{i}\right)$, where $\left(\phi_{i}\right)_{0}(x)=\left(\phi_{i}(x), \rho(x, \theta)\right)$. 
Proof. It follows directly from Definition 1.5 that the above conditions are necessary for the $\alpha$-outer conjugacy. So, we have to prove their sufficiency. Let $p=p\left(\theta_{i}\right)>$ 0 (if $p\left(\theta_{i}\right)=0$, the proof is simplified). Set $g=g\left(\theta_{i}, \phi_{i}\right)$ and $H_{0}=r\left(\Gamma, \alpha_{0}\right)$. Let $\tilde{\alpha}_{i}$ be the extension of $\alpha_{i}$ on $\Gamma_{\theta_{i}}$ with values in $G(g, p)$ (see $\left.\S 1\right), i=1,2$. Then Proposition 1.4 implies the existence of pairs $\left(g_{i}, s_{i}\right) \in G \times \mathbb{R}$ such that $\left(\phi_{i}(x)-g_{i}, \rho\left(x, \theta_{i}\right)-s_{i}\right) \in H_{0}$ for a.e. $x$. Hence $\left(g+p g_{i}, p s_{i}\right) \in H_{0}$. It is easy to see that the cocycles $\left(\tilde{\alpha}_{i}\right)_{0}=\left(\tilde{\alpha}_{i}, \rho\right)$ are regular and

$$
\begin{aligned}
r\left(\Gamma_{\theta_{i}},\left(\tilde{\alpha}_{i}\right)_{0}\right)=\left\{\left(j g_{i}+h, j, j s_{i}+s\right) \in G(g, p) \times \mathbb{R} \text { for all }(h, s)\right. & \in H_{0} \\
\text { and } j & =0, \ldots, p-1\},
\end{aligned}
$$

$i=1,2$. Since $\Psi_{\alpha_{0}}\left(\theta_{i},\left(\phi_{i}\right)_{0}\right)=W_{\alpha_{0}}\left(g_{i}, s_{i}\right)$ and $\Psi_{\alpha_{0}}\left(\theta_{1},\left(\phi_{1}\right)_{0}\right)=\Psi_{\alpha_{0}}\left(\theta_{2},\left(\phi_{2}\right)_{0}\right)$, we have $\left(g_{1}-g_{2}, s_{1}-s_{2}\right) \in H_{0}$. Thus, $r\left(\Gamma_{\theta_{1}},\left(\tilde{\alpha}_{1}\right)_{0}\right)=r\left(\Gamma_{\theta_{2}},\left(\tilde{\alpha}_{2}\right)_{0}\right)$. It is known that the groups $\Gamma_{\theta_{i}}$ are a.f., $i=1,2[\mathrm{CFW}]$. Therefore, $\left(\Gamma_{\theta_{1}}, \tilde{\alpha}_{1}\right)$ and $\left(\Gamma_{\theta_{2}}, \tilde{\alpha}_{2}\right)$ are weakly equivalent [BG3], [GS1]. To complete the proof we need only to apply the following lemma.

Lemma 3.2. Let $\theta_{1}$ and $\theta_{2}$ be compatible with a (not necessary regular) cocycle $\alpha$, $p\left(\theta_{1}\right)=p\left(\theta_{2}\right)$, and $g\left(\theta_{1}, \phi_{1}\right)=g\left(\theta_{2}, \phi_{2}\right)$ for some functions $\phi_{i} \in \alpha\left(\theta_{i}\right)$. If $\left(\Gamma_{\theta_{1}}, \tilde{\alpha}_{1}\right)$ and $\left(\Gamma_{\theta_{2}}, \tilde{\alpha}_{2}\right)$ are weakly equivalent, then $\theta_{1}$ and $\theta_{2}$ are $\alpha$-outer conjugate.

Proof. Let $p=p\left(\theta_{i}\right)>0$ (if $p=0$, the proof is similar). Then there exist an automorphism $\zeta$ of $(X, \mu)$ and a function $\psi: X \rightarrow G(g, p)$ such that $\zeta^{-1}\left[\Gamma_{\theta_{2}}\right] \zeta=$ $\left[\Gamma_{\theta_{1}}\right]$ and

$$
\zeta \circ \tilde{\alpha}_{1}\left(x, \gamma_{2}\right)=-\psi(x)+\tilde{\alpha}_{2}\left(x, \gamma_{2}\right)+\psi\left(\gamma_{2} x\right)
$$

for every $\gamma_{2} \in \Gamma_{\theta_{2}}$ at a.e. $x$. We assume that $\psi(x)=\left(\psi_{1}(x), 0\right)$ for some function $\psi_{1}: X \rightarrow G$. This can be obtained by left multiplying $\zeta$ with a certain automorphism $t \in\left[\Gamma_{\theta_{2}}\right]$ (see Lemma 2.2 from [BG2]). Let us substitute automorphisms $\gamma \in \Gamma$ and then $\theta_{2}$ in (8). We have: $\zeta \in N[\Gamma], \zeta^{-1} \theta_{2} \zeta=\theta_{1} \gamma_{0}$ for some $\gamma_{0} \in[\Gamma]$ and

$$
\zeta \circ \alpha(x, \gamma)=-\psi_{1}(x)+\alpha(x, \gamma)+\psi_{1}(\gamma x)
$$

for a.e. $x$, i.e. $\zeta \in D(\Gamma, \alpha)$. This proves the lemma.

If $\alpha_{0}$ is regular then $\alpha$ and $\rho$ are also regular. The converse statement is not true [BG3]. If $\Gamma$ is of type $I I I$ and $\rho$ is a regular cocycle, then $r(\Gamma, \rho)$ is equal to $\{n \cdot \log \lambda \mid n \in \mathbb{Z}\}$ for some $0<\lambda<1$ or to $\mathbb{R}$. Then $\Gamma$ is said to be of type $I I I_{\lambda}$ or $I I I_{1}$ respectively. If $\rho$ is not regular, $\Gamma$ is said to be of type $I I I_{0}$. Let $p \in \mathbb{Z}_{+}$. In [Da1] a pair $(\Gamma, \alpha)$ is constructed in such a way that $\alpha_{0}$ is regular and there exists an uncountable family of $\alpha$-compatible automorphisms which are pairwise not $\alpha$-outer conjugate. Moreover, $\Gamma$ is of type $I I I_{1}$ and the outer period of every element from the family is equal to $p$. Hence, these automorphisms are pairwise outer conjugate.

Our purpose now is to describe the group of approximately inner automorphisms $\mathrm{Cl}_{d_{\alpha}}([\Gamma])$, i.e. the $d_{\alpha}$-closure of $[\Gamma]$. By $H$ we denote the projection of $H_{0}=$ $r\left(\Gamma, \alpha_{0}\right) \subset G \times \mathbb{R}$ to $\mathbb{R}$. First, we formulate an auxiliary lemma. Its proof for a type $I I_{1}$ group $\Gamma$ is given in Example 1.2. A similar argument proves the lemma in the general case (see [Da1]).

Lemma 3.3. Let $\alpha_{0}$ be a regular cocycle and $\theta$ an $\alpha$-compatible automorphism. Then there exist a d.s. $\left(X_{1}, \mu_{1}, \Gamma_{1}\right)$, a cocycle $\alpha_{1} \in Z^{1}\left(X_{1} \times \Gamma_{1}, G\right)$, and an automorphism $\theta_{1} \in D\left(\Gamma_{1}, \alpha_{1}\right)$ such that 
(i) $\left(\Gamma_{1}, \alpha_{1}\right)$ is weakly equivalent to $(\Gamma, \alpha)$,

(ii) $\left(\alpha_{1}\right)_{0}$ takes its values in a countable dense subgroup of $H_{0}$ and we have $\left(\alpha_{1}\right)_{0}(x, Q)=0$ for some ergodic automorphism $Q \in\left[\Gamma_{1}\right]$,

(iii) $p\left(\theta_{1}\right)=p(\theta), g\left(\theta_{1}, \varphi_{1}\right)=g(\theta, \varphi)$, and $\Psi_{\left(\alpha_{1}\right)_{0}}\left(\theta_{1},\left(\varphi_{1}\right)_{0}\right)=\Psi_{\alpha_{0}}\left(\theta, \varphi_{0}\right)$ for some functions $\varphi \in \alpha(\theta)$ and $\varphi_{1} \in \alpha\left(\theta_{1}\right)$,

(iv) the class $\alpha_{1}\left(\theta_{1}\right)$ consists of constant a.e. functions and we have $\rho\left(x, \theta_{1}\right)=$ const,

(v) if $\Psi_{\alpha_{0}}\left(\theta, \varphi_{0}\right)=W_{\alpha_{0}}(h, 0)$ for some $h \in G$, then $\theta_{1}$ can be chosen to be $\mu_{1}$ preserving,

(vi) for every $s \in \mathrm{Cl}(H)$ there exist an automorphism $\zeta \in \mathrm{Cl}_{d_{\alpha_{1}}}\left(\left[\Gamma_{1}\right]\right)$ and element $h \in G$ with $\Psi_{\left(\alpha_{1}\right)_{0}}(\zeta, \psi)=W_{\alpha_{0}}(h, s)$, where $\psi=0$.

Theorem 3.4. If $\alpha_{0}$ is regular, then $\mathrm{Cl}_{d_{\alpha}}([\Gamma])=D(\Gamma, \alpha) \cap \mathrm{Cl}_{d}([\Gamma])$.

Proof. We put $L=G \times \mathrm{Cl}(H)$. Let $\theta$ be a $\alpha$-compatible automorphism and $\Psi_{\alpha_{0}}\left(\theta, \varphi_{0}\right) \in W_{\alpha_{0}}(L)$ for some $\varphi \in \alpha(\theta)$. It follows from (i) and (vi) of Lemma 3.3 that there exists an automorphism $\zeta \in \mathrm{Cl}_{d_{\alpha}}([\Gamma])$ such that $\Phi_{\alpha_{0}}(\theta \zeta)=W_{\alpha_{0}}(G, 0)$. Apply Lemma 3.3 to the triple $(\Gamma, \alpha, \theta \zeta)$. Then by virtue of Theorem 3.1 one can deduce that the isomorphism $\eta: X \rightarrow X_{1}$ taking $(\Gamma, \alpha)$ to $\left(\Gamma_{1}, \alpha_{1}\right)$ takes automorphism $\theta \zeta t$ to $\theta_{1}$ with some transformation $t \in[\Gamma]$. Consider a sequence $\left\{\xi_{n}\right\}_{n=1}^{\infty}$ of $\Gamma_{1}$-arrays on $\left(X_{1}, \mu_{1}\right)$ such that (see [BG2, Theorem 2.1] and [BG3, Lemma 3.11]:

(i) $\xi_{n}=\left(A_{n-1}\left(i_{n-1}\right), \Xi_{n}, A_{n}(),. \gamma_{n}(.,).\right)$ for some $i_{n-1} \in \Xi_{n-1}$ (we assume that $\left.A_{0}\left(i_{0}\right)=X_{1}\right)$

(ii) $\left[\bigcup_{n=1}^{\infty} \mathcal{G}\left(\xi_{1} \times \cdots \times \xi_{n}\right)\right]=\left[\Gamma_{1}\right]$;

(iii) $\bigcup_{n=1}^{\infty} \mathcal{P}\left(\xi_{1} \times \cdots \times \xi_{n}\right)=\mathcal{B}_{1} \bmod 0$;

(iv) for every $\gamma \in \mathcal{G}\left(\xi_{1} \times \cdots \times \xi_{n}\right)$ and an atom $c \in \mathcal{P}\left(\xi_{1} \times \cdots \times \xi_{n}\right)$ we have $\left(\alpha_{1}\right)_{0}(x, \gamma)=$ const at a.e. $x \in X_{1}$.

Since $\theta_{1}$ and $Q$ preserve $\mu_{1}$ (see Lemma 3.3(ii) and (v)), we have that for every $n \in \mathbb{N}$ there exists an automorphism $q_{n} \in[Q]$ such that $\theta_{1} A_{n}\left(i_{n}\right)=q_{n} A_{n}\left(i_{n}\right)[\mathrm{Kr}]$, [HO]. Put

$$
r_{n}(x)=\theta_{1} \gamma \theta_{1}^{-1} q_{n} \gamma^{-1} x, \quad x \in \gamma A_{n}\left(i_{n}\right),
$$

where $\gamma=\gamma_{1}\left(j_{1}, i_{1}\right) \gamma_{2}\left(j_{2}, i_{2}\right) \cdots \gamma_{n}\left(j_{n}, i_{n}\right), j_{k} \in \Xi_{k}, k=1, \ldots, n$. Then the automorphisms $r_{n} \in\left[\Gamma_{1}\right], n \in \mathbb{N}$. A routine calculation shows that $\left(\alpha_{1}\right)_{0}\left(x, r_{n}\right)=0$ and $d\left(r_{n}, \theta_{1}\right) \rightarrow 0$ as $n \rightarrow \infty$. Therefore $\theta_{1} \in \mathrm{Cl}_{d_{\alpha_{1}}}\left(\left[\Gamma_{1}\right]\right)$. Hence $\theta \zeta t \in \mathrm{Cl}_{d_{\alpha}}([\Gamma])$. By virtue of Theorem 2.4, $\theta$ is an approximately inner automorphism. So,

$$
\left\{\theta \in D(\Gamma, \alpha) \mid \Psi_{\alpha_{0}}\left(\theta, \varphi_{0}\right) \in W_{\alpha_{0}}(L) \quad \text { for some } \varphi \in \alpha(\theta)\right\} \subset \mathrm{Cl}_{d_{\alpha}}([\Gamma]) .
$$

Theorem 2.8 implies the converse inclusion.

Let $\Gamma$ be of type $I I, \theta \in N[\Gamma]$, and $\lambda$ be a $\Gamma$-invariant $\mu$-equivalent measure. Then one has $\log \frac{d \lambda \circ \theta}{d \lambda}(x)=\bmod \theta$ at a.e. $x$ for some positive number $\bmod \theta[\mathrm{CK}]$, $[\mathrm{HO}]$. Hence, if $\Gamma$ is of

(i) type $I I$, then $\mathrm{Cl}_{d_{\alpha}}([\Gamma])=\{\theta \in D(\Gamma, \alpha) \mid \bmod \theta=0\}$;

(ii) type $I I I_{1}$, then $\mathrm{Cl}_{d_{\alpha}}([\Gamma])=D(\Gamma, \alpha)$;

(iii) type $I I I_{\lambda}, 0<\lambda<1$, then $\mathrm{Cl}_{d_{\alpha}}([\Gamma])=\left\{\theta \in D(\Gamma, \alpha) \mid \Phi_{\alpha_{0}}=W_{\alpha_{0}}(G, 0)\right\}$.

Since $\alpha_{0}$ is regular, we see that $\Gamma$ is not of type $I I I_{0}$ and the map $\tau_{0}$ in diagram (2) is onto. By virtue of the results from [BG1], $[\mathrm{HO}],[\mathrm{H}]$ the theorem is completely proved. 


\section{Automorphisms COMPATIBLE With NONREGUlAR COCYClES. FreE ASSOCIATED ACTIONS}

4.0. It is known that the Mackey action $W_{\alpha}$ of $G$ (associated with $(\Gamma, \alpha)$ ) is free if and only if $r(\Gamma, \alpha)=0$. Let $\Gamma$ act freely. A cocycle $\alpha$ is called

(i) transient if the $\alpha$-skew product action $V_{\alpha}$ of $\Gamma$ (see $\left.\S 1.2\right)$ is of type $I$, i.e. the $V_{\alpha}(\Gamma)$-orbit partition of $\left(X \times G, \mu \times \mu_{G}\right)$ is measurable,

(ii) recurrent if it is not transient.

4.1. Throughout this section we assume that $\alpha$ or, equivalently, $\alpha_{0}$ [BG3] is transient. If (and only if) $\Gamma$ is of type $I I$, then one can assume that $\nu$ is $W_{\alpha}(G)$-invariant. Note that $\nu$ may be finite or infinite ( $\sigma$-finite) regardless of $\Gamma$ being of type $I I_{1}$ or $I I_{\infty}$

Theorem 4.1. Let an automorphism $\zeta \in C\left(W_{\alpha}\right)$. If $\Gamma$ is of type $I I_{\infty}$ or $I I I$, then there exist an automorphism $\theta \in D(\Gamma, \alpha)$ and a function $\varphi \in \alpha(\theta)$ such that $\zeta=\Psi_{\alpha}(\theta, \varphi)$. The same is true when $\Gamma$ is of type $I I_{1}$ if $\zeta$ preserves the $W_{\alpha}(G)$ invariant measure $\nu$.

Proof. We shall only consider the case of continuous group $G$. If $G$ is discrete, the proof is clear. It is convenient to apply the theory of measurable equivalence relations (measurable groupoids) [M2], [R1], [R2], [FHM]. Let $\mathcal{H}$ be a measurable equivalence relation generated by $W_{\alpha}$ and $\mathcal{F}$ be the transitive equivalence relation on the circle $\mathbb{T}$. By $E$ we denote a full lacunar countable section for $W_{\alpha}$ and by $\nu_{E}$ the projection of $\nu$ on $E$ along $W_{\alpha}$-orbits [FHM], [R2]. Since $E$ is lacunar, there is a discrete equivalence relation $\mathcal{R}$ on it and, hence, a countable group $\Sigma$ of automorphisms on $\left(E, \nu_{E}\right)$ generating $\mathcal{R}[\mathrm{FM}]$. The map $\pi_{E}\left(\omega, W_{\alpha}(g) \omega\right)=g$ is called the return homomorphism on $E$. An automorphism of $\mathcal{H}$ corresponding to $\zeta$ will be also denoted by $\zeta$. It is known that the Cartesian product $\mathcal{R} \times \mathcal{F}$ is isomorphic to $\mathcal{H}$ [FHM, Theorem 6.4]. Therefore we shall identify them. Then there exist an inner automorphism $\tau$ of $\mathcal{H}$ (i.e. $\omega$ and $\tau \omega$ are $W_{\alpha}(G)$-equivalent for a.e. $\omega$ ) and an automorphism $\vartheta$ of $\mathcal{R}$ such that

$$
\tau \zeta=\vartheta \times \mathrm{id}
$$

(see [GS1, Theorem 2.4]). Let the homomorphism $\pi$ of $\mathcal{H}$ into $G$ be given by $\pi\left(\omega, W_{\alpha}(g) \omega\right)=g$ and let $\psi(\omega)=\pi(\omega, \tau \omega)$. Then

$$
(\tau \zeta)^{-1} \circ \pi(\omega, v)=-\psi(\omega)+\pi(\omega, v)+\psi(v)
$$

for all $(\omega, v)$ from some inessential reduction of $\mathcal{H}$. Hence there defines an element $t_{0} \in \mathbb{T}$ such that (10) is valid for a.e. $(\omega, v) \in \mathcal{R} \times\left\{\left(t_{0}, t_{0}\right)\right\}$. It follows from (9) that $\vartheta$ is compatible with $\pi_{E}$, i.e. $\pi_{E}$ and $\vartheta \circ \pi_{E}$ are cohomologous. Theorem 7.4 from $[\mathrm{FHM}]$ implies that $(\Gamma, \alpha)$ and $\left(\Sigma, \pi_{E}\right)$ are stably weak equivalent. Therefore $\vartheta$ corresponds to a $\alpha$-compatible automorphism $\theta$. A calculation implies $\Phi_{\alpha}(\theta)=$ $\zeta W_{\alpha}(G)$.

Remark 4.2. Since $\alpha$ is transient, it follows that $\Phi_{\alpha}(\theta) \neq W_{\alpha}(G)$ for any $\theta \in$ $D(\Gamma, \alpha) \backslash[\Gamma]$.

Corollary 4.3. If $\Gamma$ is of type $I I_{\infty}$ or $I I I$ then the quotient groups $D(\Gamma, \alpha) /[\Gamma]$ and $C\left(W_{\alpha}\right) / W_{\alpha}(G)$ are algebraically isomorphic. The same is true when $\Gamma$ is of type $I I_{1}$ if the $W_{\alpha}(G)$-invariant measure is finite. 
Corollary 4.4. If $\Gamma$ is of type II $\theta \in D(\Gamma, \alpha), \varphi \in \alpha(\theta)$, and $\bmod \theta=0$, then $\Psi_{\alpha}(\theta, \varphi)$ preserves the $W_{\alpha}(G)$-invariant measure.

The next statement follows straightforwardly from Theorem 4.1 and Corollary 4.3.

Theorem 4.5. Let $\Gamma$ not be of type $I I_{1}$. Two $\alpha$-compatible automorphisms $\theta_{1}$ and $\theta_{2}$ are $\alpha$-outer conjugate iff there exists an automorphism $\zeta \in C\left(W_{\alpha}\right)$ such that

$$
\Psi_{\alpha}\left(\theta_{1}, \varphi_{1}\right)=\zeta \Psi_{\alpha}\left(\theta_{2}, \varphi_{2}\right) \zeta^{-1}
$$

for some functions $\varphi_{i} \in \alpha\left(\theta_{i}\right), i=1,2$. The same is true when $\Gamma$ is of type $I I_{1}$ if $\zeta$ preserves the $W_{\alpha}(G)$-invariant measure.

Note that (11) implies $p\left(\theta_{1}\right)=p\left(\theta_{2}\right)$ and $g\left(\theta_{1}, \varphi_{1}\right)=g\left(\theta_{2}, \varphi_{2}\right)$.

Definition 4.6. An ergodic action $V$ of $G$ on $(X, \mu)$ is called $w$-rigid if the set $V(G)$ is weakly closed in $\operatorname{Aut}(X, \mu)$.

Proposition 4.7. If the action $W_{\alpha}(G)$ is w-rigid then $\mathrm{Cl}_{d_{\alpha}}([\Gamma])=[\Gamma]$.

The proof is obvious.

Example 4.8. Let $T$ be an ergodic automorphism on $(X, \mu)$ such that $C(T)=$ $\left\{T^{n} \mid n \in \mathbb{Z}\right\}$ (see, for example, [O]). Set $\alpha\left(x, T^{n}\right)=n$ for all $x \in X, n \in \mathbb{N}$. It is easy to see that the cocycle $\alpha \in Z^{1}(X \times[T], \mathbb{Z})$ is transient. Since the centralizer of $W_{\alpha}$ is trivial, the action $W_{\alpha}$ is $w$-rigid. Therefore $D(T, \alpha)=[T]$, i.e. each automorphism compatible with $\alpha$ is inner. Note that only transient cocycles can satisfy this property.

Remark 4.9. Since the commutativity of $G$ is used nowhere in Theorem 4.1, all statements of $\S 4.1$ can be easily reformulated for non-Abelian $G$.

4.2. Throughout this section (if the contrary is not stated explicitly) we assume that $\Gamma$ is an a.f. type $I I_{\infty}$ group, $\alpha$ is a nonregular recurrent cocycle, and $W_{\alpha}$ is free. Replacing if it is necessary $(\Gamma, \alpha)$ by a weakly equivalent pair we can assume that [BG3]:

A1) $(X, \mu)=(Z \times Y, \kappa \times \lambda)$ for some Lebesgue spaces $(Z, \kappa)$ and $(Y, \lambda)$;

A2) $\Gamma$ is generated by two automorphisms $S_{0}$ and $Q_{0}$ :

$$
S_{0}(z, y)=(z, S y), \quad Q_{0}(z, y)=\left(Q z, U_{z} y\right),
$$

where $S$ is an ergodic type $I I_{\infty}$ automorphism of $(Y, \lambda), \lambda$ is a $S$-invariant measure, $Q$ is an ergodic automorphism of $(Z, \kappa)$, and $Z \ni z \rightarrow U_{z}$ is a measurable field of transformations on $(Y, \lambda)$ such that $U_{z} \in N[S]$ for a.e. $z \in Z$.

A3) $\alpha$ is determined on the generators of $\Gamma$ by:

$$
\alpha\left(z, y, S_{0}\right)=0, \quad \alpha\left(z, y, Q_{0}\right)=\delta(z, Q),
$$

where $\delta \in Z^{1}(Z \times[Q], G)$ is a transient cocycle.

Moreover, since all ergodic type $I I_{\infty}$ automorphisms are orbitally equivalent, we can assume that

A4) $(Y, \lambda)=\left(Y_{1} \times \mathbb{R}, \lambda_{1} \times \sigma\right)$ and $S\left(y_{1}, t\right)=\left(T y_{1}, t+\rho\left(y_{1}, T\right)\right)$, where $T$ is an ergodic type $I I I_{1}$ automorphism of $\left(Y_{1}, \lambda_{1}\right)$, and $\sigma$ is the measure on $\mathbb{R}$ determined by $\log \frac{d \sigma}{d \lambda_{\mathbb{R}}}(t)=-t$ for all $t \in \mathbb{R}$ (remind that $\lambda_{\mathbb{R}}$ is Lebesgue measure on $\mathbb{R}$ ). 
Then the map $\pi: \mathbb{R} \rightarrow N[S]$ determined by $\pi\left(t_{1}\right)\left(y_{1}, t\right)=\left(y_{1}, t+t_{1}\right)$ is a Borel homomorphism with $\bmod \pi\left(t_{1}\right)=t_{1}$ for each $t_{1} \in \mathbb{R}$. Therefore we can assume that

A5) $U_{z}=\pi(\rho(z, Q))$ for a.e. $z$, i.e. $Q_{0}$ preserves $\mu$.

Now we can describe a structure of automorphisms compatible with $\alpha$.

Proposition 4.10. For every automorphism $\theta \in D(\Gamma, \alpha)$ there exist transformations $\tau \in[\Gamma]$ and $\vartheta \in D(Q, \delta)$ such that $\tau \theta(z, y)=\left(\vartheta z, V_{z} y\right)$ for a.e. $(z, y) \in X$, where $Z \ni z \rightarrow V_{z}$ is a measurable field of transformations on $(Y, \lambda), V_{z} \in N[S]$ and $\bmod \theta=\bmod V_{z}+\rho(z, \vartheta)$ for a.e. $z$.

Proof. Choose $\phi \in \alpha(\theta)$ and set $\zeta=\Psi_{\alpha}(\theta, \phi)$. It is straightforward that $W_{\alpha}=W_{\delta}$ (see A3)). Then by Theorem 4.1 there are $\vartheta \in D(Q, \delta)$ and $\psi \in \delta(\vartheta)$ with $\zeta=$ $\Psi_{\delta}(\vartheta, \psi)$. One can check that the formula

$$
\xi(z, y)=(\vartheta z, \pi(\rho(z, \vartheta)-\bmod \theta) y)
$$

defines an $\alpha$-compatible automorphism $\xi$ with $\bmod \xi=\bmod \theta$ and $\Psi_{\alpha}(\theta, \chi)=\zeta$ for the function $\chi(z, y)=\psi(z)$ at all $(z, y) \in X$. We put $\eta=\theta \xi^{-1}$. Then $\eta$ is a $\mu$-preserving, $\alpha$-compatible automorphism, and $\Psi_{\alpha}(\eta, v)=$ id for some function $v \in \alpha(\eta)$. We write $\eta$ as $\eta(z, y)=(A(z, y), B(z, y))$. Note that a.e. $V_{\alpha}(\Gamma)$ ergodic component is of the form: $V_{\delta}(Q)$-orbit $\times Y$. By the $V_{\delta}(Q)$-orbit of a point $(z, g) \in Z \times G$ we mean the set $\left\{\left(Q^{n} z, g+\delta(z, Q)\right) \mid n \in \mathbb{Z}\right\}$. Since $\Psi_{\alpha}(\eta, v) \omega=\omega$ for a.e. $\omega \in \Omega$, a.e. $\eta_{v}$-orbit (see $\S 1.3$ ) is contained in a $V_{\alpha}(\Gamma)$-ergodic component. Hence $A(z, y)=Q^{n(z, y)} z$ for a.e. $(z, y)$, where $n: X \rightarrow \mathbb{Z}$ is a measurable function. Let $X_{m}=\left\{(z, y) \mid A(z, y)=Q^{m}\right\}$. Then $X=\bigcup_{m \in \mathbb{Z}} X_{m}$. Since $\eta$ and $Q_{0}$ preserve $\mu$, we have

$$
\lambda\left(\left\{y \in Y \mid(z, y) \in \eta X_{m}\right\}\right)=\lambda\left(\left\{y \in Y \mid(z, y) \in Q_{0}^{m} X_{m}\right\}\right)
$$

for a.e. $z \in Z$ and each $m \in \mathbb{Z}$. Therefore there exist one-to-one maps $\tau_{m}$ : $Q_{0}^{m} X_{m} \rightarrow \eta X_{m}$ with $\tau_{m}(z, y)=\left(z, S^{l_{m}(z, y)} y\right)$ for a.e. $(z, y)$, where $l: Q_{0}^{m} X_{m} \rightarrow \mathbb{Z}$ are some measurable maps, $m \in \mathbb{Z}[\mathrm{Kr}]$, [HO]. Now we define an automorphism $\tau \in[\Gamma]$ by

$$
\tau(z, y)=Q_{0}^{-m} \tau_{m}^{-1}(z, y)
$$

for all $(z, y) \in \eta X_{m}$. Obviously, $\tau \eta(z, y)=\left(z, \widetilde{V}_{z} y\right)$ for a.e. $(z, y)$, where $Z \ni z \rightarrow \widetilde{V}_{z}$ is a measurable field of transformations on $(Y, \lambda)$. Since $\tau \eta \in N[\Gamma]$ it follows that $\tau \eta \in N\left[S_{0}\right]$. Hence, $\widetilde{V}_{z} \in N[S]$ for a.e. $z \in Z$. Thus the proposition is proved.

Remark 4.11. The $\alpha$-fundamental homomorphism $\Phi_{\alpha}: D(\Gamma, \alpha) \rightarrow C\left(W_{\alpha}\right) / W_{\alpha}(G)$ is onto.

Theorem 4.12. Two $\alpha$-compatible automorphisms $\theta_{1}$ and $\theta_{2}$ are $\alpha$-outer conjugate if and only if $p\left(\theta_{1}\right)=p\left(\theta_{2}\right), \bmod \theta_{1}=\bmod \theta_{2}$ and there exists an automorphism $\zeta \in C\left(W_{\alpha}\right)$ such that

$$
\Psi_{\alpha}\left(\theta_{1}, \varphi_{1}\right)=\zeta \Psi_{\alpha}\left(\theta_{2}, \varphi_{2}\right) \zeta^{-1}
$$

for some functions $\varphi_{i} \in \alpha\left(\theta_{i}\right), i=1,2$.

Proof. It is sufficient to prove the if part. Set $p=p\left(\theta_{i}\right)$. By Proposition 4.10 we can assume that $\theta_{i}(z, y)=\left(\vartheta_{i} z, V_{i}(z) y\right)$, where $\vartheta_{i} \in D(\theta, \delta)$ and $Z \ni z \rightarrow$ $V_{i}(z)$ are measurable fields of automorphisms from $N[S]$. It is easy to check that $\varphi_{i}(z, y)=\psi_{i}(z)$ at a.e. $(z, y) \in X$ for some functions $\psi_{i} \in \delta\left(\vartheta_{i}\right)$. Consequently, 
$g\left(\theta_{i}, \varphi_{i}\right)=g\left(\vartheta_{i}, \psi_{i}\right), i=1,2$. Then (12) implies $g\left(\theta_{i}, \varphi_{i}\right)=g\left(\vartheta_{i}, \psi_{i}\right)$ (see the remark just after Theorem 4.5). Hence one can define the extensions $\tilde{\alpha}_{i}$ of $\alpha$ on $\Gamma_{\theta_{i}}$ with values in $G \times \mathbb{Z}$ or $G(g, p)$ when $p=0$ or $p>0$ respectively, where $g=g\left(\theta_{i}, \varphi_{i}\right)$, $i=1,2$. It follows from the assumptions of the theorem that the actions associated with $\left(\Gamma_{\theta_{i}},\left(\alpha_{i}\right)_{0}\right), i=1,2$, are isomorphic. Hence $\left(\Gamma_{\theta_{1}}, \alpha_{1}\right)$ and $\left(\Gamma_{\theta_{2}}, \alpha_{2}\right)$ are weakly equivalent [BG3, Theorems 5.12 and 7.2]. To complete the proof, it remains to apply Lemma 3.2 .

Note that any condition of Theorem 4.12: $\left(p\left(\theta_{1}\right)=p\left(\theta_{2}\right), \bmod \theta_{1}=\bmod \theta_{2}\right.$, and (12)) does not follow from the other ones. By $d_{w}$ we denote a metric compatible with the weak topology on the set of all automorphisms of $(X, \mu)[\mathrm{HO}]$.

Theorem 4.13. $\mathrm{Cl}_{d_{\alpha}}([\Gamma])=\left\{\theta \in D(\Gamma, \alpha) \mid \Phi_{\alpha}(\theta) \in \mathrm{Cl}_{d_{w}}\left(W_{\alpha}(G)\right) / W_{\alpha}(G)\right.$ and $\bmod \theta=0\}$.

Proof. We only consider the case of a continuous group $G$. Assume that there are a sequence $\left\{g_{n}\right\}_{n=1}^{\infty}$ of elements from $G$ and an automorphism $\zeta \in C\left(W_{\alpha}\right)$ such that $d_{w}\left(W_{\alpha}\left(g_{n}\right), \zeta\right) \rightarrow 0$. Choose a countable dense subgroup $G_{d}$ in $G$ containing $\left\{g_{n}\right\}_{n=1}^{\infty}$. Then $W_{\alpha}\left(G_{d}\right)$ is a countable ergodic a.f. group of automorphisms on $(\Omega, \nu)$. Put $\left(X_{1}, \mu_{1}\right)=(\Omega \times Y, \nu \times \lambda)$. By $\Gamma_{1}$ we denote the group of automorphisms on $\left(X_{1}, \mu_{1}\right)$ generated by $S_{1}$ and $\gamma_{g}, g \in G_{d}$ :

$$
S_{1}(\omega, y)=(\omega, S y), \quad \gamma_{g}(\omega, y)=\left(W_{\alpha}(g) \omega, \pi\left(\rho\left(\omega, W_{\alpha}(g)\right)\right) y\right) .
$$

Define a cocycle $\alpha_{1}$ of the d.s. $\left(X_{1}, \mu_{1}, \Gamma_{1}\right)$ by

$$
\alpha_{1}\left(x_{1}, S_{1}\right)=0, \quad \alpha_{1}\left(x_{1}, \gamma_{g}\right)=g
$$

at all $x_{1} \in X_{1}$ for every $g \in G_{d}$. It is easy to see that the actions $W_{\alpha_{1}}$ and $W_{\alpha}$ of $G$ are isomorphic. Hence $(\Gamma, \alpha)$ and $\left(\Gamma_{1}, \alpha_{1}\right)$ are weakly equivalent. Consider the automorphism $\xi_{1}$ on $\left(X_{1}, \mu_{1}\right)$ :

$$
\xi_{1}(\omega, y)=(\zeta \omega, \pi(\rho(\omega, \zeta)) y)
$$

It is straightforward to check the following properties of $\xi_{1}: \xi_{1} \in D\left(\Gamma_{1}, \alpha_{1}\right)$, $\bmod \xi_{1}=0$, and $\Phi_{\alpha_{1}}\left(\xi_{1}\right)=\xi W_{\alpha_{1}}(G)$. Moreover, one can check that $\left\{\gamma_{g_{n}}\right\}_{n=1}^{\infty}$ $\alpha_{1}$-converges to $\xi_{1}$ as $n \rightarrow \infty$. Therefore $\xi_{1}$ corresponds to an automorphism $\xi \in \mathrm{Cl}_{d_{\alpha}}([\Gamma])$ so that $\bmod \xi=0$ and $\Phi_{\alpha}(\xi)=\zeta W_{\alpha}(G)$. Thus for the completion of the proof we need to establish that

$$
\mathrm{Cl}_{d_{\alpha}}([\Gamma]) \supset\left\{\theta \in D(\Gamma, \alpha) \mid \bmod \theta=0 \text { and } \Phi_{\alpha_{0}} \in W_{\alpha}(G)\right\} .
$$

Suppose that $\theta \in D(\Gamma, \alpha), \bmod \theta=0$, and there exists a function $\varphi \in \alpha(\theta)$ with $\Psi_{\alpha}(\theta, \varphi)=$ id. By Proposition 4.10 one can assume that $\theta$ is of the following form: $\theta(z, y)=\left(z, V_{z} y\right)$, where $\bmod V_{z}=0$ and $p\left(V_{z}\right)=p(\theta)$ for a.e. $z$. Choose the automorphisms $t_{n} \in[S]$ and $\eta \in N[S]$ so that $\bmod \eta=0, p(\eta)=p(\theta), t_{n} U_{z}=U_{z} t_{n}$ for all $n \in \mathbb{N}$ and a.e. $z$, and $t_{n} \rightarrow \eta$ in the metric $d[\mathrm{HO}]$ as $n \rightarrow \infty$. We put $\eta_{0}(z, y)=(z, \eta y)$ for all $(z, y) \in X$. Then $\eta_{0} \in D(\Gamma, \alpha)$. Theorem 4.12 implies that $\theta$ and $\eta_{0}$ are $\alpha$-outer conjugate. Now define an automorphism $\gamma_{n} \in[\Gamma]$ by $\gamma_{n}(z, y)=\left(z, t_{n} y\right)$ for all $(z, y) \in X, n \in \mathbb{N}$. A routine calculation shows that $\left\{\gamma_{n}\right\}_{n=1}^{\infty} \alpha$-converges to $\eta_{0}$. Since $D(\Gamma, \alpha)$ is a topological group, it follows that $\theta \in \mathrm{Cl}_{d_{\alpha}}([\Gamma])$, as desired.

Corollary 4.14. If $W_{\alpha}$ is w-rigid, then

$$
\mathrm{Cl}_{d_{\alpha}}([\Gamma])=\left\{\theta \in D(\Gamma, \alpha) \mid \Phi_{\alpha}(\theta) \in W_{\alpha}(G) \text { and } \bmod \theta=0\right\} .
$$


Remark 4.15. Theorems 4.12 and 4.13 are also true when $\Gamma$ is of type $I I_{1}$. To prove this one must only consider a countable extension $(\hat{\Gamma}, \hat{\alpha})$ of $(\Gamma, \alpha)$ and notice that if $\bmod \theta_{i}=0$ under the conditions of Theorem 4.12, then there exists an automorphism $\xi$ that $\alpha$-outer conjugates $\theta_{1}$ and $\theta_{2}$ and whose $\bmod \xi=0$.

4.3. Using Theorem 4.13, we can describe the group of approximately inner automorphisms compatible with a transient cocycle (see also Proposition 4.7).

Theorem 4.16. Let $\Sigma$ be an ergodic a.f. group of automorphisms on a Lebesgue space $(Z, \kappa)$ and $\delta$ a transient cocycle of $(Z, \kappa, \Sigma)$ with values in $G$. Then

$$
\mathrm{Cl}_{d_{\delta}}([\Sigma])=\left\{\vartheta \in D(\Sigma, \delta) \mid \Phi_{\delta}(\vartheta) \in \mathrm{Cl}_{d_{w}}\left(W_{\delta}(G)\right) / W_{\delta}(G)\right\} .
$$

Proof. Take an automorphism $Q \in[\Sigma]$ with $[\Sigma]=[Q]$. Consider the pair $(\Gamma, \alpha)$ determined by the quadruple $(Z, \kappa, Q, \delta)$ in such a way that A1)-A5) are valid (see $\S 4.2)$. Select an automorphism $\vartheta \in D(\Sigma, \delta)$ with $\Phi_{\delta}(\vartheta) \in \mathrm{Cl}_{d_{w}}\left(W_{\delta}(G)\right) / W_{\delta}(G)$. By Theorem 4.13 there are an automorphism $\theta \in \mathrm{Cl}_{d_{\alpha}}([\Gamma])$ and a sequence $\left\{\gamma_{n}\right\}_{n=1}^{\infty}$ of automorphisms from $[\Gamma]$ such that $\Phi_{\alpha}(\theta)=\Phi_{\delta}(\vartheta)$ and $d_{\alpha}\left(\gamma_{n}, \theta\right) \rightarrow 0$. In view of Proposition 4.10 and Remark 4.2 we may assume that $\theta(z, y)=\left(\vartheta z, V_{z} y\right)$ for a.e. $(z, y) \in X$. Moreover, we assume that $V_{z}=\pi(\rho(z, \vartheta))$ for a.e. $z$, since every automorphism $\eta \in N[\Gamma]$ of the form $\eta(z, y)=\left(z, V_{z}^{\prime} y\right)$ is approximately inner and compatible with $\alpha$ (see the proof of Theorem 4.13). Since $\pi(\mathbb{R}) \subseteq C(S)$ (see A4)), it follows that $\theta$ commutes with $S_{0}$. By $\mu_{1}$ we denote a probability measure on $X$ equivalent to $\mu$. Since $d\left(\gamma_{n}, \theta\right) \rightarrow 0$ as $n \rightarrow \infty$, we have

$$
\mu_{1}\left(\left\{x \mid \gamma_{n}^{-1} S_{0} \gamma_{n} x \neq S_{0} x\right\}\right) \rightarrow 0 .
$$

Lemma 4.17. For an automorphism $\gamma \in[\Gamma]$ let $B=\left\{x \in X \mid S_{0} \gamma x \neq \gamma S_{0} x\right\}$. Then there exists an automorphism $\eta \in[\Gamma]$ with $\eta S_{0}=S_{0} \eta$ and $\eta x=\gamma x$ for all $x \notin B$.

Proof. Note that $S_{0} \gamma(B)=\gamma S_{0}(B)(\bmod 0)$. Set $\eta x=\gamma x$ for all $x \notin B$. We continue the construction of $\eta$ inductively. Let

$$
C_{0}=B, \quad B_{n}=S_{0}^{-1}\left(X \backslash C_{n-1}\right) \cap C_{n-1}, \quad C_{n}=C_{n-1} \backslash B_{n} .
$$

Put $\eta x=S_{0}^{-1} \eta S_{0} x$ for all $x \in B_{n}$. If there is $N \in \mathbb{N} \cup\{\infty\}$ such that $B=\bigcup_{n=1}^{\infty} B_{n}$ $(\bmod 0)$, then the above formula determines a well-defined automorphism $\eta$ as desired. Otherwise we denote by $B_{\infty}$ the set $B \backslash \bigcup_{n=1}^{\infty} B_{n}$. One can check that it is $S_{0}$-invariant. Hence $B_{\infty}=A \times Y \bmod 0$ for some subset $A \subseteq Z, \kappa(A)>0$. Then we set $\eta x=S_{0} x$ for all $x \in B_{\infty}$. Evidently, $\eta$ is the desired automorphism.

Return to the proof of Theorem 4.16. Apply Lemma 4.17 to $\gamma_{n}$, and denote by $\eta_{n}$, $n \in \mathbb{N}$, the automorphism whose existence is asserted in the lemma. It follows from (13) that $\eta_{n} \rightarrow \theta$ in $d_{\alpha}$ as $n \rightarrow \infty$. But since $\eta_{0}$ commutes with $S_{0}$, we have

$$
\eta_{n}(z, y)=\left(q_{n} z, V_{n}(z) y\right)
$$

for a.e. $(z, y)$ and an automorphism $q_{n} \in[\Sigma]$, where $Z \ni z \rightarrow V_{n}(z)$ is a measurable field of automorphisms from $N[S]$. One can check that $q_{n} \rightarrow \vartheta$ in $d_{\delta}$. So,

$$
\mathrm{Cl}_{d_{\delta}}([\Sigma]) \supseteq\left\{\vartheta \in D(\Sigma, \delta) \mid \Phi_{\delta}(\vartheta) \in \mathrm{Cl}_{d_{w}}\left(W_{\delta}(G)\right) / W_{\delta}(G)\right\} .
$$

The inverse inclusion is obvious. Thus the theorem is proved completely. 
4.4. Now we consider an ergodic type $I I I$ a.f. group $\Gamma$ of automorphisms on $(X, \mu)$ and its cocycle $\alpha$ such that the "double" cocycle $\alpha_{0}=(\alpha, \rho)$ is recurrent and nonregular, and $W_{\alpha_{0}}$ is free. Then one can replace $(\Gamma, \alpha)$ by a weakly equivalent pair in such a way that the following five conditions are valid: A1), A2), A4) (see $\S 4.2)$,

B3) $\alpha_{0}$ is defined on the generators $S_{0}$ and $Q_{0}$ by

$$
\alpha_{0}\left(z, y, S_{0}\right)=0 \text { and } \alpha_{0}\left(z, y, Q_{0}\right)=\delta(z, Q),
$$

where $\delta=\left(\delta_{1}, \delta_{2}\right) \in Z^{1}(Z \times[Q], G \times \mathbb{R})$ is a transient cocycle [BG3, Theorem 5.6],

B5) $U_{z}=\pi\left(\rho(z, Q)-\delta_{2}(z, Q)\right)$ for all $z \in Z$.

We do not provide the proof of the next theorem, since it is a slightly modified version of Proposition 4.10 and Theorems 4.12, 4.13.

Theorem 4.18. (i) Let an automorphism $\theta$ be compatible with $\alpha$. Then there exist automorphisms $\tau \in[\Gamma]$ and $\vartheta \in D(Q, \delta)$ such that

$$
\tau \theta(z, y)=\left(\vartheta z, V_{z} y\right)
$$

for a.e. $(z, y) \in X$, where $Z \ni z \rightarrow V_{z}$ is a measurable field of automorphisms on $(Y, \lambda)$ and $V_{z} \in N[S]$ at a.e. $z$,

(ii) the $\alpha_{0}$-fundamental homomorphism $\Phi_{\alpha_{0}}: D(\Gamma, \alpha) \rightarrow C\left(W_{\alpha_{0}}\right) / W_{\alpha_{0}}(G, 0)$ is onto,

(iii) two $\alpha$-compatible automorphisms $\theta_{1}$ and $\theta_{2}$ are $\alpha$-outer compatible iff $p\left(\theta_{1}\right)=$ $p\left(\theta_{2}\right)$ and

$$
\Psi_{\alpha_{0}}\left(\theta_{1},\left(\varphi_{1}\right)_{0}\right)=\zeta \Psi_{\alpha_{0}}\left(\theta_{2},\left(\varphi_{2}\right)_{0}\right) \zeta^{-1}
$$

for some automorphism $\zeta \in C\left(W_{\alpha_{0}}\right)$ and for $\left(\varphi_{i}\right)_{0}(z)=\left(\varphi_{i}(z), \rho\left(z, \theta_{i}\right)\right)$, where $\varphi_{i} \in \alpha\left(\theta_{i}\right), i=1,2$,

(iv) $\mathrm{Cl}_{d_{\alpha}}([\Gamma])=\left\{\theta \in D(\Gamma, \alpha) \mid \Phi_{\alpha_{0}}(\theta) \in \mathrm{Cl}_{d_{w}}\left(W_{\alpha_{0}}(G, 0)\right) / W_{\alpha_{0}}(G, 0)\right\}$.

Remark 4.19. Note that even if $\theta_{1}$ and $\theta_{2}$ are outer conjugate and $\Psi_{\alpha}\left(\theta_{1}, \psi_{1}\right)=$ $\xi \Psi_{\alpha}\left(\theta_{2}, \psi_{2}\right) \xi^{-1}$ for some $\psi_{i} \in \alpha\left(\theta_{i}\right)$ and $\xi \in C\left(W_{\alpha}\right)$, then they need not be $\alpha$-outer conjugate, i.e. (14) need not be valid - one can construct a pair $(\Gamma, \alpha)$ in such a way that $\Gamma$ is of type $I I I_{1}, r(\Gamma, \alpha)=G, W_{\alpha_{0}}$ is free, and the centralizer of $W_{\alpha_{0}}$ contains nonconjugate elements [BG3, Example 7.4].

\section{Automorphisms COMPatible With NOnREgular COCYCles. NONFREE ASSOCIATED ACTIONS}

5.0. Throughout this section we assume that $\Gamma$ is a.f. and a cocycle $\alpha$ of the d.s. $(X, \mu, \Gamma)$ is such that $\alpha_{0}$ is nonregular and $W_{\alpha_{0}}$ is nonfree. All the other cases were considered before (for an a.f. group $\Gamma$ ). The stabilizer of $W_{\alpha_{0}}$ is equal to $r\left(\Gamma, \alpha_{0}\right)$ (see $\S 1.2)$.

5.1. In this subsection we assume $\Gamma$ is of type $I I_{\infty}$ (if the contrary is not stated explicitly). Then there exists a closed subgroup $H \subset G$ such that $H=r(\Gamma, \alpha)$. Replacing if necessary $(\Gamma, \alpha)$ by a weakly equivalent pair we assume that the following conditions are valid: A1), A2), A4), A5) (see $\S 4.2$ ), and

C3) $\alpha$ is determined on the generators of $\Gamma$ by

$$
\alpha\left(z, y, S_{0}\right)=\beta(y, S), \quad \alpha\left(z, y, Q_{0}\right)=\delta(z, Q),
$$


where $\beta \in Z^{1}(Y \times[S], G)$ is a regular cocycle with $r(S, \beta)=H$, and $\delta \in$ $Z^{1}(Z \times[Q], G)$ is so that the $H$-factor cocycle $\delta_{H} \in Z^{1}(Z \times[Q], G / H)$ (i.e. $\left.\delta_{H}(z, Q)=\delta(z, Q)+H\right)$ is transient (as well as $\delta$ ).

Set $(\tilde{Y}, \tilde{\lambda})=(Y, \lambda) \otimes(Y, \lambda), S_{1}=S \times \operatorname{id}, S_{2}=\operatorname{id} \times S, \tilde{\beta}\left(y, y_{1}, S_{1}\right)=\beta(y, S)$, $\tilde{\beta}\left(y, y_{1}, S_{2}\right)=0, \tilde{\pi}(t)=\mathrm{id} \times \pi(t)$ for all $\left(y, y_{1}\right) \in \tilde{Y}, t \in \mathbb{R}$. Denote by $\tilde{S}$ an ergodic automorphism of $(\tilde{Y}, \tilde{\lambda})$ such that $[\tilde{S}]$ is equal to the full group generated by $S_{1}$ and $S_{2}$. Now identifying $\tilde{Y}, \tilde{\lambda}, \tilde{S}, \tilde{\beta}, \tilde{\pi}$ with $Y, \lambda, S, \beta, \pi$ respectively we obtain a pair $(\Gamma, \alpha)$ satisfying the conditions A1), A2), C3), A5) and

C6) $\pi(t) \in D(S, \beta)$ and $\pi(t) \circ \beta=\beta$ for all $t \in \mathbb{R}$.

Lemma 5.1. Given an automorphism $\zeta \in C\left(W_{\delta_{H}}\right)$, there exist a cocycle $\delta^{\prime} \in$ $Z^{1}(Z \times[Q], G)$ and an automorphism $\vartheta \in D\left(Q, \delta^{\prime}\right)$ such that $\delta_{H}^{\prime}=\delta_{H}$ and $\Psi_{\delta_{H}^{\prime}}=$ $\zeta W_{\delta_{H}}(G / H)$.

Proof. By Theorem 4.1 there are an automorphism $\vartheta \in D\left(Q, \delta_{H}\right)$ and a function $\varphi_{H} \in \delta_{H}(\vartheta)$ so that $\zeta=\Psi_{\delta_{H}}\left(\vartheta, \varphi_{H}\right)$. Let $\sigma: G / H \rightarrow G$ be a Borel cross-section of the projection $j: G \rightarrow G / H$, i.e. $j \circ \sigma=$ id and $T$ an automorphism on $(Z, \kappa)$ such that $[T]$ equals to the full group generated - as a full group - by $Q$ and $\vartheta$.

We first assume that $p(\vartheta)=0$. Denote by $\delta_{H}^{+} \in Z^{1}(Z \times[T], G / H)$ the extension of $\delta_{H}$ to $[T]$ (see Remark 1.6) and consider the function

$$
f: Z \ni z \mapsto f(z) \stackrel{\text { def }}{=} \sigma\left(\delta_{H}^{+}(z, T)\right) .
$$

We define the cocycle $\delta^{\prime} \in Z^{1}(Z \times[Q], G)$ by setting

$$
\delta^{\prime}(z, Q)= \begin{cases}f(z)+f(T z)+\cdots+f\left(T^{l-1} z\right), & \text { if } l>0, \\ -f\left(T^{-1} z\right)-f\left(T^{-2} z\right)-\cdots-f\left(T^{l} z\right), & \text { if } l<0,\end{cases}
$$

where the integer $l=l(z)$ is determined by $Q z=T^{l} z$ for a.e. $z$. It is straightforward to check that $\delta_{H}^{\prime}=\delta_{H}$ and $\vartheta \in D\left(Q, \delta^{\prime}\right)$, as desired.

Now let $p=p(\vartheta)>0$. Consider the extension $\tilde{\delta}_{H}$ of $\delta_{H}$ on $[T]$ with values in $(G / H)\left(g_{H}, p\right)$, where $g_{H}=g\left(\vartheta, \varphi_{H}\right)$ (see $\left.\S 1.3\right)$. Set $g=\sigma\left(g_{H}\right)$. Consider the function $f_{1}: Z \rightarrow G(g, p)$ given by $f_{1}(z)=(f(z), m)$, where $m=m(z)$ is determined by $T z=Q^{n} \vartheta^{m} z, 0 \leq m<p$, for a.e. $z$. Substitute $f_{1}$ in (15) for $f$. Then (15) defines a cocycle $\delta^{\prime}$ of the d.s. $(Z, \kappa, Q)$ with values in $G \subset G(g, p)$. Evidently, $\vartheta \in D\left(Q, \delta^{\prime}\right)$.

Proposition 5.2. For any automorphism $\theta \in D(\Gamma, \alpha)$ there exist a cocycle $\delta^{\prime} \in$ $Z^{1}(Z \times Q, G)$ and transformations $\tau \in[\Gamma]$ and $\vartheta \in D\left(Q, \delta^{\prime}\right)$ such that $\delta_{H}^{\prime}=\delta_{H}$ and $\tau \theta(z, y)=\left(\vartheta z, V_{z} y\right)$ for a.e. $(z, y) \in X$, where $Z \ni z \rightarrow V_{z}$ is a measurable field of automorphisms on $(Z, \kappa)$ satisfying the conditions: $V_{z} \in D(S, \beta)$ and $\bmod \theta=$ $\bmod V_{z}+\rho(z, \vartheta)$ for a.e. $z \in Z$.

Proof. Set $\zeta=\Psi_{\alpha}(\theta, \varphi)$ for a function $\phi \in \alpha(\theta)$ and apply Lemma 5.1. The completion of the proof coincides almost literally with that of Proposition 4.10.

Notice, that the change of $\delta$ for $\delta^{\prime}$ in C3) implies that we pass from $(\Gamma, \alpha)$ to a weakly equivalent pair.

Corollary 5.3. The $\alpha$-fundamental homomorphism

$$
\Phi_{\alpha}: D(\Gamma, \alpha) \rightarrow C\left(W_{\alpha}\right) / W_{\alpha}(G)
$$

is onto. 
Theorem 5.4. Two $\alpha$-compatible automorphisms $\theta_{1}$ and $\theta_{2}$ are $\alpha$-outer conjugate iff $p\left(\theta_{1}\right)=p\left(\theta_{2}\right), \bmod \theta_{1}=\bmod \theta_{2}, g\left(\theta_{1}, \varphi_{1}\right)=g\left(\theta_{2}, \varphi_{2}\right)$, and $\Psi_{\alpha}\left(\theta_{1}, \varphi_{1}\right)=$ $\zeta \Psi_{\alpha}\left(\theta_{2}, \varphi_{2}\right) \zeta^{-1}$ for some functions $\varphi_{i} \in \alpha\left(\theta_{i}\right)$ and an automorphism $\zeta \in C\left(W_{\alpha}\right)$.

This theorem is an analogue of Theorems 3.1 and 4.12 and can be proved in a similar way.

The following example shows that the condition $g\left(\theta_{1}, \varphi_{1}\right)=g\left(\theta_{2}, \varphi_{2}\right)$ in Theorem 5.4 may not be omitted (cf. Theorem 4.12).

Example 5.5. Let ( $\Gamma, \alpha)$ satisfy A1), A2), C3), A4), and A5), the automorphism $Q$ preserve $\kappa$, and $U_{z}=$ id for a.e. $z$. Select automorphisms $\eta_{i} \in D(S, \beta)$ such that the following conditions are satisfied: $\bmod \theta_{i}=0, p\left(\theta_{i}\right)=p$, and $g\left(\eta_{1}, \varphi_{1}\right) \neq g\left(\eta_{2}, \varphi_{2}\right)$ for all $\varphi_{i} \in \beta\left(\eta_{i}\right), i=1,2$ (see, for instance, Example 1.2). Put $\theta_{i}(z, y)=\left(z, \eta_{i} y\right)$ for all $(z, y) \in X, G=\mathbb{Z} \times \mathbb{Z}$, and $H=\mathbb{Z} \times\{0\}$. Then $p\left(\theta_{i}\right)=p, \quad \bmod \theta_{i}=0$, $\Phi_{\alpha}\left(\theta_{i}\right)=W_{\alpha}(G)$, but $g\left(\theta_{1}, \psi_{1}\right) \neq g\left(\theta_{2}, \psi_{2}\right)$ for every $\psi_{i} \in \alpha\left(\theta_{i}\right), i=1,2$. Hence, $\theta_{1}$ and $\theta_{2}$ are not $\alpha$-outer conjugate.

Remark 5.6. Theorem 4.13 is also true for pairs $(\Gamma, \alpha)$ described in the beginning of the section and its proof may be obtained in a similar way.

Remark 5.7. Theorems 5.4 and 4.13 are also true when $\Gamma$ is of type $I I_{1}$ (cf. Remark 4.15).

5.2. Now let $\Gamma$ be of type $I I I$. Set up $H_{0}=r\left(\Gamma, \alpha_{0}\right)$. Replacing if it is necessary $(\Gamma, \alpha)$ by a weakly equivalent pair, one may assume that [BG3, Theorems 6.4 and 7.5]:

D1) $(X, \mu)=(Z, \kappa) \otimes(y, \lambda)$,

D2) $\Gamma$ is generated by the automorphisms $S_{0}$ and $Q_{0}$ :

$$
S_{0}(z, y)=(z, S y), \quad Q_{0}(z, y)=\left(Q z, U_{z} y\right)
$$

where $S$ and $Q$ are ergodic automorphisms on $(Y, \lambda)$ and $(Z, \kappa)$ respectively and $Z \ni z \rightarrow U_{z}$ is a measurable field of automorphisms such that $U_{z} \in N[S]$ for a.e. $z$,

D3) $\alpha$ is determined on the generators of $\Gamma$ by

$$
\alpha_{0}\left(z, y, S_{0}\right)=\beta_{0}(y, S), \quad \alpha_{0}\left(z, y, Q_{0}\right)=\delta(z, Q),
$$

where the cocycles $\beta \in Z^{1}(Y \times[S], G)$ and $\delta=\left(\delta_{1}, \delta_{2}\right) \in Z^{1}(Z \times[Q], G \times \mathbb{R})$ are such that $\beta_{0}$ is regular, $r\left(S, \beta_{0}\right)=H_{0}$, and $\delta_{H_{0}} \in Z^{1}(Z \times[Q], G \times \mathbb{R})$ (see C3)) is transient (as well as $\delta$ ),

D4) there is a continuous homomorphism $\pi: \mathbb{R} \rightarrow D(S, \beta)$ such that $\pi(t) \circ \beta_{0}=\beta_{0}$ for all $t \in \mathbb{R}$ and $U_{z}=\pi\left(\rho(z, Q)-\delta_{2}(z, Q)\right)$ for a.e. $z \in Z$.

The specific form of $H_{0}$ as above ensures that $S$ is of type $I I$ or $I I I_{\lambda}(0<\lambda \leq 1)$.

Theorem 5.8. (i) For an automorphism $\theta \in D(\Gamma, \alpha)$ there exist a cocycle $\delta^{\prime} \in$ $Z^{1}(Z \times[Q], G \times \mathbb{R})$ and transformations $\tau \in[\Gamma]$ and $\vartheta \in D\left(Q, \delta^{\prime}\right)$ such that $\delta_{H_{0}}^{\prime}=$ $\delta_{H_{0}}$ and $\tau \theta(z, y)=\left(\vartheta z, V_{z} y\right)$ for a.e. $(z, y) \in X$.

(ii) Two $\alpha$-compatible automorphisms $\theta_{1}$ and $\theta_{2}$ are $\alpha$-outer conjugate if and only if $p\left(\theta_{1}\right)=p\left(\theta_{2}\right), g\left(\theta_{1}, \varphi_{1}\right)=g\left(\theta_{2}, \varphi_{2}\right)$, and

$$
\Psi_{\alpha_{0}}\left(\theta_{1},\left(\varphi_{1}\right)_{0}\right)=\zeta \Psi_{\alpha_{0}}\left(\theta_{2},\left(\varphi_{2}\right)_{0}\right) \zeta^{-1}
$$

for some functions $\varphi_{i} \in \alpha\left(\theta_{i}\right), i=1,2$, and an automorphism $\zeta \in C\left(W_{\alpha_{0}}\right)$.

Moreover, the statements (ii) and (iv) of Theorem 4.18 are also true for this pair $(\Gamma, \alpha)$. 


\section{Cocycles Compatible With Every Element of NORMalizer}

6.0. Let $\alpha$ be a cocycle of an a.f. automorphism group $\Gamma$ with values in an Abelian l.c.s.c. group $G$.

Definition 6.1. Let us say that $\alpha$ has property $A$ (or is $A$-cocycle) if $D(\Gamma, \alpha)=$ $N[\Gamma]$.

The main goal of this section is to prove the following statement announced in [Da2]:

Theorem 6.2. $\alpha$ has property A iff one of the following conditions is satisfied (it depends on the type of $\Gamma)$ :

(i) (for $\Gamma$ of type $I I) \alpha$ is a coboundary,

(ii) (for $\Gamma$ of type $\left.I I I_{\lambda}, 0<\lambda \leq 1\right) \alpha_{0}=\alpha \times \rho$ is regular, i.e. $W_{\alpha_{0}}(G \times \mathbb{R})$ is transitive, and $r\left(\Gamma, \alpha_{0}\right)=\bigcup_{s \in r(\Gamma, \rho)}(l(s), s)$ for some continuous homomorphism $l: r(\Gamma, \rho) \rightarrow G$,

(iii) (for $\Gamma$ of type $\left.I I I_{0}\right)$ a.e. $W_{\alpha_{0}}(G, 0)$-ergodic component is isomorphic to the shiftwise action of $G$ on $\left(G, \mu_{G}\right)$ and the natural map $C\left(W_{\alpha_{0}}\right) \rightarrow C\left(W_{\rho}\right)$ is onto.

So, weakly equivalent classes of $A$-cocycles are in one-to-one correspondence with elements of $G$ when $\Gamma$ is of type $I I I_{\lambda}, 0<\lambda<1$, and with the graphs of continuous homomorphisms from $\mathbb{R}$ into $G$ when $\Gamma$ is of type $I I I_{1}$. Every $A$-cocycle is a coboundary for $\Gamma$ of type $I I$, and for type $I I I_{1}$ if, additionally, $G$ is discrete. If $\Gamma$ is of type $I I I_{0}$, then $W_{\alpha_{0}}(G \times \mathbb{R})$ is a free action for every $A$-cocycle.

The if part of Theorem 6.2 is obvious for (i) and (ii) and directly follows from Proposition 4.10, Theorem 4.18(i) and [BG3]. In the next two subsections we produce the proof of the only if part which seems to be more difficult.

6.1. First of all we consider the most important cases of regular and transient cocycles.

Lemma 6.3. Let $p \in \mathbb{N}, M$ be a countable subset of $G, Y_{k}=\{0,1, \ldots, p-1\}$, $\nu_{k}(0)=\cdots=\nu_{k}(p-1)=1 / p,(Y, \nu)=\prod_{k=1}^{\infty}\left(Y_{k}, \nu_{k}\right), \Delta$ the automorphisms group generated by $\delta_{k}, k \in \mathbb{N}:\left(\delta_{k} y\right)_{j}=y_{j}+\delta_{k j}(\bmod p)$, and $\left\{g_{k}\right\}_{k=1}^{\infty}$ be a sequence of elements of $M$, in which every element of $M$ occurs infinitely many times. Set

$$
\beta\left(y, \delta_{k}\right)= \begin{cases}g_{k}, & \text { if } y_{k} \neq p-1, \\ -(p-1) g_{k}, & \text { otherwise. }\end{cases}
$$

Then the cocycle $\beta \in Z^{1}(Y \times \Delta, G)$ is regular and $r(\Delta, \beta)$ is the smallest closed subgroup of $G$ containing $M$.

This lemma is a slightly modified version of Theorem 3.2 from [BG3] and can be proved in a similar way.

Proposition 6.4. Let $\Gamma$ be of type II and $\alpha$ a regular A-cocycle. Then $\alpha$ is a coboundary.

Proof. Let $M$ be a countable dense subset of $r(\Gamma, \alpha)$ and $p=2$. Apply Lemma 6.2. Since the group $r(\Gamma, \alpha)=r(\Delta, \beta)$ is a complete invariant of the stably weak equivalence for regular cocycles, we can identify $(\Gamma, \alpha)$ with $(\Delta, \beta)$. Consider an automorphism $\theta \in N[\Delta]$ given by $(\theta y)_{j}=y_{j}+1(\bmod p)$. Since $\theta$ is compatible with 
$\alpha$, there exists a function $\varphi: X \rightarrow G$ such that

$$
(-1)^{y_{k}+1} g_{k}=-\varphi(y)+(-1)^{y_{k}} g_{k}+\varphi\left(\delta_{k} y\right)
$$

for all $k \in \mathbb{N}$ at a.e. $y \in Y$. Hence $(-1)^{y_{k}} 2 g_{k}=\varphi(y)-\varphi\left(\delta_{k} y\right)$, i.e. $2 \alpha$ is a coboundary. It follows then $2 r(\Gamma, \alpha) \subseteq r(\Gamma, 2 \alpha)=\{0\}$. Thus, $r(\Gamma, \alpha)$ is a periodic group and every nonzero element of $r(\Gamma, \alpha)$ has the order 2 . Now let $p=3$. Reasoning similarly, we obtain that the cocycle $\epsilon$ of $\Delta$ given by

$$
\epsilon\left(y, \delta_{k}\right)= \begin{cases}0, & \text { if } y_{k}=0 \\ 3 g_{k}, & \text { if } y_{k}=1, \\ -3 g_{k}, & \text { if } y_{k}=2\end{cases}
$$

is a coboundary. It is easy to deduce from this that $3 r(\Gamma, \alpha)=\{0\}$. Hence, $r(\Gamma, \alpha)=\{0\}$, i.e. $\alpha$ is a coboundary.

Proposition 6.5. Let $\alpha$ be a transient cocycle. Then $\alpha$ doesn't have the A-property.

Proof. Suppose the contrary. Without loss of generality we may assume that $\Gamma$ is not of type $I I_{1}$. Otherwise consider the countable extension $(\tilde{\Gamma}, \tilde{\alpha})$ of $(\Gamma, \alpha)$. Select two automorphisms $\theta_{i} \in N[\Gamma] \backslash[\Gamma]$ such that $\bmod \theta_{i}=0, i=1,2$, and the commutator $\left[\theta_{1}, \theta_{2}\right] \notin[\Gamma]$ (see [GS1, Theorem 5.4]). Since $\theta_{i} \in \mathrm{Cl}_{d}([\Gamma])$ (see $[\mathrm{HO}]$ ),

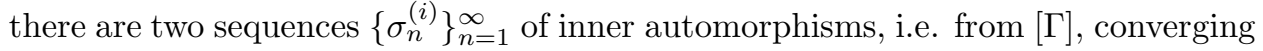
to $\theta_{i}$ in the metric $d, i=1,2$. Since the natural embedding $D(\Gamma, \alpha) \rightarrow N[\Gamma]$ is continuous and onto, then by a version of Banach's theorem on closed graphs and Theorem 2.3 it is a homeomorphism. Hence, $\sigma_{n}^{(i)} \rightarrow \theta_{i}$ in $d_{\alpha}$ as $n \rightarrow \infty, i=1,2$. Choose the corresponding sequences of functions $\varphi_{n}^{(i)} \in \alpha\left(\sigma_{n}^{(i)}\right)$ and $\varphi_{i} \in \alpha\left(\theta_{i}\right)$, $i=1,2, n \in \mathbb{N}$, as in Definition 2.1. In view of Theorem $2.8 \Psi_{\alpha}\left(\sigma_{n}^{(i)}, \varphi_{n}^{(i)}\right) \rightarrow$ $\Psi_{\alpha}\left(\theta_{i}, \varphi_{i}\right)$ as $n \rightarrow \infty, i=1,2$, in the weak topology $d_{w}$ on $C\left(W_{\alpha}(G)\right)$. Since $\Psi_{\alpha}\left(\sigma_{n}^{(i)}, \varphi_{n}^{(i)}\right) \in W_{\alpha}(G)$, then $\Psi_{\alpha}\left(\theta_{i}, \varphi_{i}\right) \in \mathrm{Cl}_{d_{w}}\left(W_{\alpha}(G)\right)$ which is an Abelian group. Therefore, $\left[\Psi_{\alpha}\left(\theta_{1}, \varphi_{1}\right), \Psi_{\alpha}\left(\theta_{2}, \varphi_{2}\right)\right]=$ id. On the other hand, since $\alpha$ is transient and $\left[\theta_{1}, \theta_{2}\right] \notin[\Gamma]$, we have by Remark 4.2 that $\Psi_{\alpha}\left(\left[\theta_{1}, \theta_{2}\right], \varphi\right) \notin W_{\alpha}(G)$ for any $\varphi \in \alpha\left(\left[\theta_{1}, \theta_{2}\right]\right)$, a contradiction.

Remark 6.6. It follows from the proof that $\mathrm{Cl}_{d}([\Gamma]) \neq \mathrm{Cl}_{d_{\alpha}}([\Gamma])$ for any transient $\alpha$.

Corollary 6.7. Let $T$ be an ergodic automorphism. Then there exists an automorphism $\theta \in N[T]$ such that $\theta t \notin C(T)$, i.e. $[\theta t, T] \neq \mathrm{id}$, for any transformation $t \in[T]$.

Proof. Consider the d.s. $(X, \mu, T)$ and its transient cocycle $\alpha$ with values in $\mathbb{Z}$ given by $\alpha\left(x, T^{n}\right)=n$. Then by Corollary 4.3 we can identify two groups $D(T, \alpha) /[T]$ and $C(T) /\left\{T^{n}\right\}_{n}$ and then apply Proposition 6.5.

The authors do not know any other method to prove the above very natural statement.

Lemma 6.8. Let two automorphisms $\xi_{1}$ and $\xi_{2}$ belong to $N[\Gamma]$ for a type II ergodic a.f. group $\Gamma$ and $\left[\xi_{1}, \xi_{2}\right] \in[\Gamma]$. Set

$$
D^{\xi_{1}, \xi_{2}}(\Gamma, \alpha)=\left\{\xi \in D(\Gamma, \alpha) \mid\left[\xi, \xi_{i}\right] \in[\Gamma] \text { for } i=1,2\right\} .
$$


Then $D^{\xi_{1}, \xi_{2}}(\Gamma, \alpha)$ is a Polish group with respect to the metric $\tilde{d}_{\alpha}$ given by

$$
\tilde{d}_{\alpha}(\zeta, \eta)=d_{\alpha}(\zeta, \eta)+\sum_{i=1}^{2} d_{u}\left(\left[\zeta^{-1} \eta, \xi_{i}\right], 1\right)
$$

and $\mathrm{Cl}_{\tilde{d}_{\alpha}}([\Gamma])=\mathrm{Cl}_{d_{\alpha}}([\Gamma]) \cap D^{\xi_{1}, \xi_{2}}(\Gamma, \alpha)$.

This statement is a generalization of Lemma 3.9 from [BG1]. We do not give its proof here since it can be obtained in a similar manner.

Lemma 6.9. Let the assumptions of Lemma 6.8 be valid.

(i) If $\alpha$ is transient, then $D^{\xi_{1}, \xi_{2}}(\Gamma, \alpha) \neq D^{\xi_{1}, \xi_{2}}(\Gamma, \rho)$.

(ii) If $\alpha$ is regular and $D^{\xi_{1}, \xi_{2}}(\Gamma, \alpha)=D^{\xi_{1}, \xi_{2}}(\Gamma, \rho)$, then $\alpha$ is a coboundary.

Proof. The proof of (i) is almost the same as that of Proposition 6.5 but Lemma 6.8 should be used instead of Theorem 2.3.

(ii) Consider the cocycle $\beta \in Z^{1}(X \times X \times \Gamma \times \Gamma, G)$ that is given by $\beta\left(x_{1}, x_{2}, \gamma_{1} \times \gamma_{2}\right)=\alpha\left(x_{1}, \gamma_{1}\right)$. There is an isomorphism $\eta:(X, \mu) \rightarrow(X \times X, \mu \times \mu)$ such that the following conditions are satisfied: $\eta[\Gamma] \eta^{-1}=[\Gamma \times \Gamma], \eta \circ \alpha=\beta$, and $\eta \xi_{i} \eta^{-1}=\left(\mathrm{id} \times \xi_{i}\right) t_{i}$ for some automorphisms $t_{i} \in[\Gamma \times \Gamma], i=1,2$. This can be proved by the same arguments as in Theorem 3.1. If $\alpha$ is not a coboundary, then by Proposition 6.3 there exists an automorphism $\theta \in N[\Gamma] \backslash D(\Gamma, \alpha)$. Therefore the automorphism $\theta \times \mathrm{id} \notin D(\Gamma \times \Gamma, \beta)$ and commutes with $\mathrm{id} \times \xi_{i}, i=1,2$. Then $\eta^{-1}(\theta \times \mathrm{id}) \eta \in D^{\xi_{1}, \xi_{2}}(\Gamma, \rho) \backslash D^{\xi_{1}, \xi_{2}}(\Gamma, \alpha)$.

Remark 6.10. $\mathrm{Cl}_{\tilde{d}_{\alpha}}([\Gamma]) \neq \mathrm{Cl}_{\tilde{d}_{\rho}}([\Gamma])$ for any transient $\alpha$.

Proof of Theorem 6.2(i). Suppose the contrary. By Propositions 6.4 and $6.5 \alpha$ is nontransient and nonregular. Without loss of generality we may assume that $W_{\alpha}(G)$ is free (otherwise consider the quotient cocycle $\alpha^{\prime}=\alpha+r(\Gamma, \alpha)$ with values in $G / r(\Gamma, \alpha))$. But then one can suppose that the conditions A1)-A5) from $\S 4.2$ are valid. For every automorphism $\vartheta \in N[Q]$ consider the transformation $\xi \in N[\Gamma]$ given by $\xi(z, y)=(\vartheta z, \pi(\rho(z, \vartheta)))$. Since $\xi \in D(\Gamma, \alpha)$, it follows that $\vartheta \in D(Q, \delta)$. Hence $\delta$ has the $A$-property, a contradiction.

(ii) Let $\lambda<1$. Then we can suppose that $\Gamma$ is generated by two automorphisms of $(X, \mu)$ : an ergodic $S$ of type $I I_{\infty}$ and $\xi \in N[S]$ with $\bmod \xi=-\log \lambda$. Obviously, $D^{\xi, 1}(S, \rho) \subseteq N[\Gamma]$. Denote by $\alpha^{\prime}$ the restriction of $\alpha$ on $[S]$. Since $D(\Gamma, \alpha)=N[\Gamma]$, we have $D^{\xi, 1}(S, \rho) \subseteq D(\Gamma, \alpha)$ and, hence, $D^{\xi, 1}(S, \rho)=D^{\xi, 1}\left(S, \alpha^{\prime}\right)$. By the same method as in (i) one can prove using Lemma 6.8 that $\alpha^{\prime}$ is a coboundary. Therefore, replacing $\alpha$ by a $S$-cohomologous cocycle we can assume that $\alpha(x, S)=0$ for a.e. $x$. Since $S$ is ergodic, $\alpha(x, \xi)=h$ for an element $h \in G$ at a.e. $x$. Hence, $\alpha_{0}$ is regular and $r\left(\Gamma, \alpha_{0}\right)=\bigcup_{n \in \mathbb{Z}}(n h,-n \log \lambda)$, as desired.

Now consider the case $\lambda=1$. Then we can suppose that $\Gamma$ is generated by three automorphisms of $(X, \mu)$ : an ergodic $S$ of type $I I_{\infty}$ and $\xi_{1}, \xi_{2} \in N[S]$ such that $\rho_{i}=\bmod \xi_{i}, i=1,2$, are rationally independent reals. By the above reasoning one can obtained that $\alpha_{0}$ is regular and

$$
r\left(\Gamma, \alpha_{0}\right)=\mathrm{Cl}\left(\bigcup_{n, m \in \mathbb{Z}}\left(h_{1} n+h_{2} m, n \rho_{1}+m \rho_{2}\right)\right)
$$

for some $h_{i} \in G, i=1,2$. Since $\rho_{1}$ and $\rho_{2}$ are arbitrary (rationally independent) reals, the natural projection of $r\left(\Gamma, \alpha_{0}\right)$ on $\mathbb{R}$ is onto. So, it remains to prove that 
$r\left(\Gamma, \alpha_{0}\right) \cap(G \times\{0\})=\{(0,0)\}$. Let $H_{0}$ be a countable dense subgroup of $r\left(\Gamma, \alpha_{0}\right)$, $\left\{\left(g_{k}, s_{k}\right)\right\}_{k=1}^{\infty}$ a sequence of elements of $H_{0}$ in which every element of $H_{0}$ appears infinitely many times, $Y_{k}=\{0,1\}, \nu_{k}(0)=\left(1+e^{s_{k}}\right)^{-1}, \nu_{k}(1)=e^{s_{k}} /\left(1+e^{s_{k}}\right)$, $(Y, \nu)=\prod_{k=1}^{\infty}\left(Y_{k}, \nu_{k}\right), \Delta$ the same group as in Lemma 6.3, and $\beta$ the cocycle of $(Y, \nu, \Delta)$ given by $\beta\left(y, \delta_{k}\right)=(-1)^{y_{k}} g_{k}$ for all $y \in Y, k \in \mathbb{N}$. Then $\beta_{0}$ is regular and $r\left(\Delta, \beta_{0}\right)=r\left(\Gamma, \alpha_{0}\right)$ [BG3, Theorem 3.2]. If there are two elements $g_{1}, g_{2} \in G$ such that $\left(g_{1}, 0\right)$ and $\left(g_{2}, 0\right) \in H_{0}$, then there exist two sequences $\left\{k_{i}\right\}_{i},\left\{m_{i}\right\}_{i}$ of positive integers such that $\alpha\left(y, \delta_{k_{i}}\right)=(-1)^{y_{k_{i}}} g_{1}, \alpha\left(y, \delta_{m_{i}}\right)=(-1)^{y_{m_{i}}} g_{2}$, and $\nu_{k_{i}}(j)=\nu_{m_{i}}(j)=\frac{1}{2}, i \in \mathbb{N}, j=1,2$. Put

$$
(\theta y)_{j}= \begin{cases}y_{m_{i}}, & \text { if } j=k_{i} \\ y_{k_{i}}, & \text { if } j=m_{i} \\ y_{j}, & \text { otherwise }\end{cases}
$$

We see that the transformation $\theta$ preserves $\nu$. Since $\theta \delta_{m_{i}} \theta^{-1}=\delta_{k_{i}}, \theta \delta_{k_{i}} \theta^{-1}=\delta_{m_{i}}$ for $i \in \mathbb{N}$, and $\theta \delta_{k} \theta^{-1}=\delta_{k}$ for all $k \neq k_{i}, m_{i}$, it follows that $\theta \in N[\Delta]$. The pairs $(\Gamma, \alpha)$ and $(\Delta, \beta)$ are weakly equivalent [BG3] and therefore $\theta$ is compatible with $\beta$. So, there is a measurable function $\varphi: Y \rightarrow G$ such that the following conditions are satisfied:

$$
\begin{aligned}
(-1)^{y_{k_{i}}} g_{2} & =-\varphi(y)+(-1)^{y_{k_{i}}} g_{1}+\varphi\left(\delta_{k_{i}} y\right), \\
(-1)^{y_{m_{i}}} g_{1} & =-\varphi(y)+(-1)^{y_{m_{i}}} g_{2}+\varphi\left(\delta_{m_{i}} y\right), \\
0 & =-\varphi(y)+\varphi\left(\delta_{k} y\right) \quad \text { if } k \neq k_{i}, m_{i}, \text { for all } i \in \mathbb{N} .
\end{aligned}
$$

Consider the cocycle $\beta^{\prime} \in Z^{1}(Y \times \Delta, G)$ given by

$$
\beta^{\prime}\left(y, \delta_{k}\right)= \begin{cases}(-1)^{y_{k_{i}}}\left(g_{2}-g_{1}\right), & \text { if } k=k_{i} \\ (-1)^{y_{m_{i}}}\left(g_{1}-g_{2}\right), & \text { if } k=m_{i}, \\ 0, & \text { otherwise }\end{cases}
$$

Then $\beta_{0}^{\prime}$ is regular and $\left(g_{1}-g_{2}, 0\right) \in r\left(\Delta, \beta_{0}^{\prime}\right)$. According to (16) $\beta_{0}^{\prime}$ is a coboundary and therefore $g_{1}=g_{2}$.

6.2. In this section the last part of Theorem 6.2 will be handled. To this end we need to generalize some results of the previous section on nonergodic d.s.

Let $S_{0}$ be a nonergodic conservative aperiodic automorphism on $(X, \mu)$ and $\mu(X)=1$. We can assume that $(X, \mu)=(Z, \kappa) \times(Y, \nu)$ and $S_{0}(z, y)=\left(z, S_{z} y\right)$ for a measurable field $Z \ni z \rightarrow S_{z}$ of ergodic automorphisms on $(Y, \nu)$ [HO]. Without loss of generality we may suppose that $S_{z}$ is not of type $I I_{1}$ for all $z \in Z$. Denote by $\mathcal{P}$ the following equivalence relation on $X:\left(z_{1}, y_{1}\right) \sim\left(z_{2}, y_{2}\right)$ if $z_{1}=z_{2}$. Let $\operatorname{Aut}_{\mathcal{P}}(X, \mu)$ be the group of all automorphisms on $(X, \mu)$ which preserve a.e. $\mathcal{P}$-class fixed $(\bmod 0)$. Set $N_{\mathcal{P}}\left[S_{0}\right]=N\left[S_{0}\right] \cap \operatorname{Aut}_{\mathcal{P}}(X, \mu)$. Then $\theta \in N_{\mathcal{P}}\left[S_{0}\right]$ if and only if $\theta(z, y)=\left(z, \theta_{z} y\right)$ at a.e. $(z, y)$ for some measurable field $Z \ni z \rightarrow \theta_{z} \in N\left[S_{z}\right]$ of automorphisms on $(Y, \nu)$. For every cocycle $\alpha \in Z^{1}\left(X \times S_{0}, G\right)$ there exists a measurable field of cocycle $Z \ni z \rightarrow \alpha_{z} \in Z^{1}\left(Y \times\left[S_{z}\right], G\right)$ such that $\alpha\left(z, y, S_{0}\right)=\alpha_{z}\left(y, S_{z}\right)$ for a.e. $(z, y) \in X$. Put $D_{\mathcal{P}}\left(S_{0}, \alpha\right)=D\left(S_{0}, \alpha\right) \cap N_{\mathcal{P}}\left[S_{0}\right]$. Then $\theta \in D_{\mathcal{P}}\left(S_{0}, \alpha\right)$ if and only if $\theta_{z} \in D\left(S_{z}, \alpha_{z}\right)$ and there is a measurable function $\varphi: X \rightarrow G$ such that $\varphi_{z}=\varphi(z,.) \in \alpha_{z}\left(\theta_{z}\right)$ for a.e. $z \in Z$.

The next statement follows from Theorem 2.4. 
Lemma 6.11. $D_{\mathcal{P}}\left(S_{0}, \alpha\right)$ is a Polish group with respect to the metric $d_{\alpha}^{\mathcal{P}}$ given by

$$
d_{\alpha}^{\mathcal{P}}\left(\theta^{(1)}, \theta^{(2)}\right)=\int_{Z} d\left(\theta_{z}^{(1)}, \theta_{z}^{(2)}\right) d \kappa(z) .
$$

If $\alpha$ is a coboundary then we write $d^{\mathcal{P}}$ for $d_{\alpha}^{\mathcal{P}}$.

Since the ergodic components of $W_{\alpha}(G)$ are in one-to-one correspondence with $W_{\alpha_{z}}(G), z \in Z$, the formula

$$
W_{\alpha}(G)=\int_{Z} W_{\alpha_{z}}(G) d \kappa(z)
$$

has an obvious sense.

Definition 6.12. A cocycle $\alpha$ of a d.s. $\left(X, \mu, S_{0}\right)$ is called

(i) transient if $\alpha_{z}$ is transient at a.e. $z \in Z$;

(ii) regular if $\alpha_{z}$ is regular at a.e. $z \in Z$;

(iii) $H$-regular for a closed subgroup $H$ of $G$ if $\alpha$ is regular and $r\left(S_{z}, \alpha_{z}\right)=H$ at a.e. $z \in Z$.

The following two statements are "nonergodic" analogues of Propositions 6.4 and 6.5.

Proposition 6.13. Let $S_{0}$ be of type $I I_{\infty}$, i.e. $S_{z}$ be of this type for a.e. $z \in Z, \alpha$ a H-regular cocycle, and $D_{\mathcal{P}}\left(S_{0}, \alpha\right)=N_{\mathcal{P}}\left[S_{0}\right]$. Then $\alpha$ is a coboundary.

Proof. Since a.a. pairs $\left(S_{z}, \alpha_{z}\right)$ are mutually weakly equivalent we can suppose that $S_{0}(z, y)=(z, R y)$ and $\alpha\left(z, y, S_{0}\right)=\beta(y, R)$ at a.e. $(z, y) \in X$ for some ergodic $I I_{\infty}$ transformation $R$ of $Y$ and a cocycle $\beta \in Z^{1}(Y \times[R], G)$. It easy to see that $\beta$ has the $A$-property. Then by Proposition 6.4 it is a coboundary and so is $\alpha$.

Proposition 6.14. Let $\alpha$ be a transient cocycle of a nonergodic d.s. $\left(X, \mu, S_{0}\right)$. Then $D_{\mathcal{P}}\left(S_{0}, \alpha\right) \neq N_{\mathcal{P}}\left[S_{0}\right]$.

Proof. Suppose the contrary. Let $\left(X_{s t}, \mu_{s t}, \Gamma_{s t}\right)$ be an ergodic d.s. of type $I I_{\infty}$. Then there exists a measurable field of isomorphisms $Z \ni z \rightarrow P_{z}: Y \rightarrow Y \times X_{s t}$ such that the measures $P_{z} \circ \nu$ and $\nu \times \mu_{s}$ are equivalent and $P_{z}\left[S_{z}\right] P_{z}^{-1}=\left[S_{z} \times \Gamma_{s t}\right]$ for a.e. $z \in Z[\mathrm{HO}],[\mathrm{Kr}]$. Take two automorphisms $\theta_{s t}^{(i)}$ of $\left(X_{s t}, \mu_{s t}\right)$ such that $\theta_{s t}^{(i)} \in \mathrm{Cl}_{d}\left(\left[\Gamma_{s t}\right]\right) \backslash\left[\Gamma_{s t}\right], i=1,2$, and $\left[\theta_{s t}^{(1)}, \theta_{s t}^{(2)}\right] \notin\left[\Gamma_{s t}\right]$. Consider two transformations of $(X, \mu)$ given by

$$
\theta^{(i)}(z, y)=\left(z, P_{z}^{-1}\left(\theta_{s t}^{(i)} \times \mathrm{id}\right) P_{z} y\right), \quad i=1,2 .
$$

It is easy to see that $\theta^{(i)} \in \mathrm{Cl}_{d^{\mathcal{P}}}\left(\left[S_{0}\right]\right) \backslash\left[S_{0}\right]$ and $\left[\theta^{(1)}, \theta^{(2)}\right] \notin\left[S_{0}\right]$. Moreover, $\theta_{z}^{(i)} \in \mathrm{Cl}_{d_{z}}\left(\left[S_{z}\right]\right) \backslash\left[S_{z}\right]$ and $\left[\theta_{z}^{(1)}, \theta_{z}^{(2)}\right] \notin\left[S_{z}\right]$ for a.e. $z$, where $d_{z}$ is the Polish metric on $N\left[S_{z}\right]$. Take $\varphi^{(i)} \in \alpha\left(\theta^{(i)}\right), i=1,2$. Since

$$
\Psi_{\alpha}\left(\theta^{(i)}, \varphi^{(i)}\right)=\int_{Z} \Psi_{\alpha}\left(\theta_{z}^{(i)}, \varphi_{z}^{(i)}\right) d \kappa(z) \in C\left(W_{\alpha}\right), \quad i=1,2,
$$

it remains only to apply the reasoning of the proof of Proposition 6.5.

Theorem 6.15. Let $S_{0}$ be aperiodic conservative transformation of type $I I$ and $\alpha$ a cocycle of $S_{0}$ such that $r\left(S_{z}, \alpha_{z}\right)=H$ at a.e. $z$ for a closed subgroup $H$ in $G$. If $D_{\mathcal{P}}\left(S_{0}, \alpha\right)=N_{\mathcal{P}}\left[S_{0}\right]$, then $\alpha$ is a coboundary. 
Proof. By virtue of Propositions 6.13 and 6.14 we can assume that $\alpha$ is nontransient and nonregular. Then one can establish the following decomposition of $\left(X, \mu, S_{0}, \alpha\right)$ (as in [BG3] for an ergodic $S_{0}$ ):

A1) $(Y, \nu)=\left(Y_{1}, \nu_{1}\right) \times\left(Y_{2}, \nu_{2}\right)$;

A2) $S_{0}$ is orbit equivalent to the automorphism group generated by $S_{10}$ and $S_{20}$ :

$$
\begin{aligned}
& S_{10}\left(z, y_{1}, y_{2}\right)=\left(z, y_{1}, T y_{2}\right), \\
& S_{20}\left(z, y_{1}, y_{2}\right)=\left(z, S_{z}^{(2)} y_{1}, U_{z, y_{1}} y_{2}\right),
\end{aligned}
$$

where $T$ is an ergodic type $I I_{\infty}$ transformation of $\left(Y_{2}, \nu_{2}\right), Z \times Y_{1} \ni \rightarrow U_{z, y_{1}}$ is a measurable field of automorphisms from $N[T]$, and $Z \ni z \rightarrow S_{z}^{(2)} \in$ $\operatorname{Aut}\left(Y_{1}, \nu_{1}\right)$ is a measurable field of ergodic transformations;

A3) a cocycle $\alpha$ is determined by

$$
\alpha\left(z, y_{1}, y_{2}, S_{10}\right)=\beta\left(y_{2}, T\right), \quad \alpha\left(z, y_{1}, y_{2}, S_{20}\right)=\delta_{z}\left(y_{1}, S_{z}^{(2)}\right),
$$

where $\beta$ is a regular cocycle, $r(T, \beta)=H$, and

$$
Z \ni z \rightarrow \delta_{z} \in Z^{1}\left(Y_{1} \times\left[S_{z}^{(2)}\right], G\right)
$$

is a measurable field of transient cocycles.

Consider the d.s. $\left(Z \times Y_{1}, \kappa \times \nu_{1}, \tilde{S}\right)$, where the transformation $\tilde{S}$ is given by $\tilde{S}\left(z, y_{1}\right)=\left(z, S_{z}^{(2)} y_{1}\right)$. Let its cocycle $\delta$ be determined by $\delta\left(z, y_{1}, \tilde{S}\right)=\delta_{z}\left(y_{1}, S_{z}^{(2)}\right)$. Then every automorphism $\tilde{\theta}$ from $N_{\mathcal{P}}[\tilde{S}]$ can be extended to an automorphism $\theta \in N_{\mathcal{P}}\left[S_{0}\right]$ by the following formula:

$$
\theta\left(z, y_{1}, y_{2}\right)=\left(z, \theta_{z} y_{1}, \pi\left(\log \frac{d \nu_{1} \circ \theta_{z}}{d \nu_{1}}\left(y_{1}\right)\right) y_{2}\right),
$$

where $\pi: \mathbb{R} \rightarrow N[\Gamma]$ is a continuous homomorphism such that $\bmod \pi(t)=-t$ for all $t \in \mathbb{R}$. Therefore, if $H=\{0\}$ then $D_{\mathcal{P}}(\tilde{S}, \delta)=N_{\mathcal{P}}[\tilde{S}]$ and by Proposition $6.14 \delta$ is a coboundary. If $H \neq\{0\}$, consider the factor-cocycle $\alpha_{H}$ given by $\alpha_{H}\left(x, S_{0}\right)=$ $\alpha\left(x, S_{0}\right)+H$. By the above arguments $\alpha_{H}$ is a coboundary. It follows $\alpha$ is $H$-regular. Hence, by Proposition $6.13 \alpha$ is a coboundary.

Note that Theorem 6.15 generalizes Theorem 6.2(i), where only ergodic transformations have been considered. Similarly, the following statement which we formulate here without proof is a generalization of Theorem 6.2(ii).

Theorem 6.16. Let $S_{0}$ be an aperiodic conservative transformation of type $I I I_{\lambda}$, $0<\lambda \leq 1$, and $\alpha$ a cocycle of $S_{0}$ such that $r\left(S_{z},\left(\alpha_{z}\right)_{0}\right)=H_{0}$ at a.e. $z$ for a closed subgroup $H_{0} \subseteq G \times \mathbb{R}$. If $D_{\mathcal{P}}\left(S_{0}, \alpha\right)=N_{\mathcal{P}}\left[S_{0}\right]$, then $\alpha$ is $H_{0}$-regular and $H_{0}$ is of the same form as in Theorem 6.2(ii).

Now fix an automorphism $Q_{0}$ from $N\left[S_{0}\right]$. It follows $Q_{0}(z, y)=\left(Q z, U_{z} y\right)$ for a.e. $(z, y) \in X$, where $Q \in \operatorname{Aut}(Z, \kappa)$ and $Z \ni z \rightarrow U_{z} \in N\left[S_{z}\right]$ is a measurable field of automorphisms on $(Y, \nu)$. Set up $D_{\mathcal{P}}^{Q_{0}}\left(S_{0}, \alpha\right)=\left\{\theta \in D_{\mathcal{P}}\left(S_{0}, \alpha\right) \mid\left[\theta, Q_{0}\right] \in\left[S_{0}\right]\right\}$.

Lemma 6.17. Let $S_{0}$ be an aperiodic conservative transformation of type $I I_{\infty}$. Then $D_{\mathcal{P}}^{Q_{0}}\left(S_{0}, \alpha\right)$ is a Polish group with respect to the metric $\hat{d}$ given by

$$
\hat{d}(\zeta, \eta)=d_{\alpha}^{\mathcal{P}}(\zeta, \eta)+d_{u}\left(\left[\zeta^{-1} \eta, Q_{0}\right], 1\right)
$$

and $\mathrm{Cl}_{\hat{d}}\left(\left[S_{0}\right]\right)=\mathrm{Cl}_{d_{\alpha}^{\mathcal{P}}}\left(\left[S_{0}\right]\right) \cap D_{\mathcal{P}}^{Q_{0}}\left(S_{0}, \alpha\right)$. 
The proof of this statement is routine and therefore we omit it here (see its close analogue in [BG1, Lemma 3.9]). The following proposition we also formulate without proof since it is an analogue of Lemma 6.9 (see also the proof of Theorem 6.2(i), (ii)).

Proposition 6.18. Let $S_{0}$ be an aperiodic conservative transformation of type II $I_{\infty}$. If $D_{\mathcal{P}}^{Q_{0}}\left(S_{0}, \alpha\right)=D_{\mathcal{P}}^{Q_{0}}\left(S_{0}, \rho\right)$, then $\alpha$ is a coboundary.

Proof of Theorem 6.2(iii). Since $\Gamma$ is of type $I I I_{0}$, we can suppose that $\Gamma$ is orbitally equivalent to the automorphisms group generated by an aperiodic conservative transformation $S_{0}$ of type $I I_{\infty}$ and an automorphism $Q_{0} \in N\left[S_{0}\right]$ such that the function $f(z)=\rho(z, Q)+\bmod U_{z}>\epsilon$ at a.e. $z$ for some $\epsilon>0[\mathrm{Kr}]$, [HO]. Consider the restriction $\alpha^{\prime}$ of $\alpha$ on $\left[S_{0}\right]$ and apply Proposition 6.18. Then $\alpha^{\prime}$ is a coboundary. Hence, replacing $\alpha$ by a $S_{0}$-cohomologous cocycle we obtain $\alpha\left(x, S_{0}\right)=0$. Moreover, $\alpha\left(z, y, Q_{0}\right)=a(z)$ at a.e. $(z, y)$ for a function $a: Z \rightarrow G$. A cocycle $\delta^{\prime} \in Z^{1}(Z \times[Q], G \times \mathbb{R})$ given by

$$
\delta^{\prime}(z, Q)=\alpha_{0}\left(z, y, Q_{0}\right)=(a(z), f(z))
$$

is transient since its second part is transient. It follows that $W_{\alpha_{0}}$ has free transitive (mod 0) components. The natural map $C\left(W_{\alpha_{0}}\right) \rightarrow C\left(W_{\rho}\right)$ is onto by Theorem 4.1, [H, Section 3], and commutative diagram (2).

Note, that both conditions in Theorem 6.2(iii) are independent.

\section{REFERENCES}

[BG1] S. I. Bezuglyi and V. Ya. Golodets, Groups of measure space transformations and invariants of outer conjugation for automorphisms from normalizers of type III full groups, J. Funct. Anal. 60 (1985), 341-369. MR 86e:46052

[BG2] Outer conjugacy for actions of countable amenable groups on a measure space, Izv. Akad Nauk SSSR Ser. Mat. 50 (1986), 643-660 (Russian); English transl. in. Math. Izv. 29 (1987), 1-18. MR 88d:46115

[BG3] Weak equivalence and structures of cocycles of an ergodic automorphism, Publ. RIMS, Kyoto Univ. 27 (1991), 577-625. MR 93a:28017

[BGD] S. I. Bezuglyi, V. Ya. Golodets, and A. I. Danilenko, Extensions of 1-cocycles of dynamical systems on normalizer elements, Dokl. Akad. Nauk UkrSSR Ser. A 1988, no. 2, 3-5 (Russian). MR 90h:28016

[CK] A. Connes and W. Krieger, Measure space automorphisms, the normalizers of their full groups, and approximate finiteness, J. Funct. Anal. 24 (1977), 336-352. MR 56:3246

[CFW] A. Connes, J. Feldman, and B. Weiss, An amenable equivalence relation is generated by a single transformation, Ergod. Theory and Dyn. Systems 1 (1981), 431-450. MR 84h:46090

[Da1] A. I. Danilenko, Ergodic dynamical systems, their cocycles, and automorphisms compatible with cocycles, Ph.D. thesis, Kharkov State University, 1991 (Russian).

[Da2] - On cocycles compatible with normalizers of full groups of measure space transformations, Dokl. Akad. Nauk Ukraine 1994, no. 7, 14-17. MR 95k:28039

[Da3] - The topological structure of Polish groups and groupoids of measure space transformations, Publ. RIMS Kyoto Univ. 31 (1995), 913-940. CMP 96:06

[FM] J. Feldman and C. C. Moore, Ergodic equivalence relations, cohomology, and von Neumann algebras. I, Trans. Amer. Math. Soc. 234 (1977), 289-324. MR 58:28261a

[FHM] J. Feldman, P. Hahn, and C. C. Moore, Orbit structure and countable sections for actions of continuous groups, Adv. in Math. 28 (1978), 186-230. MR 58:11217

[FSZ] J. Feldman, C. E. Sutherland, and R. Zimmer, Subrelations of ergodic equivalence relations, Ergod. Theory and Dyn. Syst. 9 (1989), 239-269. MR 91c:28020

[G] V. Ya. Golodets, Description of representations of anticommutation relations, Russian Uspekhi Mat. Nauk 24 (1969), 3-64 (Russian); English transl. in. Russian Math. Surveys 24 (1969). 
[GD] V. Ya. Golodets and A. I. Danilenko, Ergodic actions of Abelian groups isomorphic to joint actions with themselves, Preprint 4-91, FTINT AN UkrSSR, Kharkov, 1991.

[GS1] V. Ya. Golodets and S. D. Sinel'shchikov, Outer conjugacy for actions of continuous amenable groups, Publ. RIMS, Kyoto Univ. 23 (1987), 737-769. MR 89c:46087

[GS2] Classification and structure of cocycles of amenable ergodic equivalence relations, J. Funct. Anal. 121 (1994), 455-485. MR 95h:28020

[H] T. Hamachi, The normalizer group of an ergodic automorphism of type III and the commutant of an ergodic flow, J. Funct. Anal. 40 (1981), 387-403. MR 82m:46070

[HO] T. Hamachi and M. Osikawa, Ergodic groups of automorphisms amd Krieger's theorems, Sem. Math. Sci. Keio Univ., 1981. MR 84d:46099

[K] A. A. Kirillov, Dynamical systems, factors, and group representations, Uspekhi Matem. Nauk 22, no. 5, (1967), 67-80 (Russian). MR 36:347

[Kr] W. Krieger, On ergodic flows and isomorphism of factors, Math. Ann. 24 (1977), 336352. MR 54:3430

[M1] G. W. Mackey, Infinite dimensional group representations, Bull. Amer. Math. Soc. 69 (1963), 628-686. MR 45:2095

[M2] Ergodic theory and virtual groups, Math. Ann. 166 (1966), 187-207. MR 34:1444

[O] D. S. Ornstein, On the root problem in ergodic theory, Proc. 6th Berkeley Symposium on Mathematical Statistics and Probability, University of California Press, 1967, pp. 347356. MR 53:3259

[R1] A. Ramsay, Virtual groups and group actions, Adv. in Math. 6 (1971), 253-322. MR 43:7590

[R2] $\quad$, Topologies for measured groupoids, J. Funct. Anal. 47 (1982), 314-343. MR 83k:22014

[S] K. Schmidt, Cocycles of ergodic transformation groups, Macmillan Company of India, Ltd, Delhi, 1977. MR 58:28262

[Z] R. Zimmer, Extension of ergodic group actions, Illinois J. Math. 20 (1976), 373-409. MR 53:13522

Department of Mechanics and Mathematics, Kharkov State University, Freedom Square 4, Kharkov, 310077, Ukraine

E-mail address: danilenko@ilt.kharkov.ua

Mathematics Department, Institute for Low Temperature Physics, Lenin Avenue 47, KHARKOV, 310164, UKRAINE

E-mail address: golodets@ilt.kharkov.ua 